\title{
Surface elasticity revisited in the context of second strain gradient theory
}

\author{
Farzaneh Ojaghnezhad ${ }^{a}$, Hossein M. Shodja ${ }^{a, b, *}$ \\ ${ }^{a}$ Department of Civil Engineering, Sharif University of Technology, P.O. Box 11155-4313, \\ Tehran, Iran \\ ${ }^{b}$ Institute for Nanoscience and Nanotechnology, Sharif University of Technology, P.O. Box \\ 11155-9161, Tehran, Iran
}

\begin{abstract}
Surface/interface stresses, when notable, are closely associated with a surface/interface layer in which the interatomic bond lengths and charge density distribution differ remarkably from those of the bulk. The presence of such topographical defects as edges and corners amplifies the noted phenomena by large amounts. If the principal features of interest are such studies as the physics and mechanics of evolving microscopic-/nanoscopic-interfaces and the behavior of nano-sized structures which have a very large surface-to-volume ratio, traditional continuum theories cease to hold. It is for the treatment of such problems that augmented continuum approaches like second strain gradient and surface elasticity theories have been developed by Mindlin (1965) and Gurtin and Murdoch (1975), respectively. In the mathematical framework of the former theory, the surface effect is explicitly revealed through surface characteristic length and modulus of cohesion, whereas within the
\end{abstract}

\footnotetext{
${ }^{*}$ Corresponding author

Email address: shodja@sharif.edu (Hossein M. Shodja)
} 
latter theory, which views the bulk material and its complementary surface as separate interacting entities, the critical role of surfaces/interfaces is directly incorporated through the introduction of the notions of tangential surface strain tensor, surface stress tensor, and surface elastic modulus tensor into the formulation. In the realm of the experimentations, evaluation of the above-mentioned surface parameters poses serious difficulties. One of the objectives of the current study is to provide a remedy as how to calculate, not only these parameters, but also Mindlin's bulk characteristic lengths as well as Lame constants with the aids of first principles density functional theory (DFT). To this end, surface elasticity is reformulated by maintaining the first and second gradients of the strain tensor for the bulk; as a result two new key equations are obtained. One of these equations is an expression for the net surface stress, needed to relate the surface parameters in surface elasticity to the Mindlin's second gradient theory parameters. The other equation is for the total elastic energy which is utilized to find an analytical expression for the surface energy. The available data on surface relaxation obtained experimentally and computationally are in good correspondence with the results of the current theory. Moreover, employing the present theory, an estimate for the effective elastic constants of films with infinite extension is provided.

Keywords: second strain gradient theory, elastic surface, surface stress, surface elastic constants, effective elastic constants, surface relaxation 


\section{Introduction}

Efforts towards the design and manufacturing of miniaturized devices in modern technologies as well as the description of the phenomena associated with the physics and mechanics of the evolving microscopic-/nanoscopic-interfaces have acquired a close examination of the topography of the three dimensional nano-sized elastic media for such defects as surfaces/interfaces, edges, and corners, respectively, referred to as the two, one, and zero dimensional defects. The equilibrium positions of the atoms located in the close vicinity of such defects significantly differ from the positions they would have when they were well into the bulk. Therefore, the mechanical properties of a nano-structure, in which the interior atoms are only a few lattice parameters away from the boundary, are significantly affected by its surface (Cammarata and Sieradzki, 1989); presence of edges and corners on the boundary imposes additional disturbance in the displacement field of the interior atoms. In fact, as

pointed out by Cammarata and Sieradzki (1989), surface stresses cause the surface atoms to displace from their equilibrium positions they would have occupied if they were well within the bulk. The phenomenon of surface relaxation has been previously studied both computationally and experimentally. Rosato et al. (1989) have employed tight-binding (TB) to study surface relaxation of some transition metals. Kuk and Feldman (1984) studied surface relaxation of $\mathrm{Ag}(110)$ through high-energy ion scattering method. A manifestation of this phenomenon is the creation of a surface layer in which the interatomic bond lengths and charge density distribution 
are quite different from those of the bulk.

Factually, continuum equations based on classical elasticity are limited to wave lengths and bodily dimensions large enough compared with the lattice parameter of the material. The idea of the characterization of the atomic structure of elastic solids through the augmentation of classical theory of elasticity has initiated with the early work of Cauchy (1851) who suggested that the local state of stress at any given field point within the three dimensional elastic solid not only depends on the displacement of the point but also is affected by the displacements of those in its vicinity. Based on this concept and the subsequent works of Voigt (1887, 1894) along this line of thought, various gradient theories have been proposed in the literature. Cauchy's continuum theory for a periodic, non-centrosymmetric, and isotropic solid can be generalized to account for the solid's anisotropy response by considering infinite gradients of strain in the potential energy density. The resulting continuum field contains the same information as the equivalent equations deduced directly from crystal lattice theory (Mindlin, 1972). Factually, consideration of the surface effect has been one of the most important stimuli in the development of augmented and higher order continuum theories which were brought into focus by many researchers, mainly, in the period of about 1960 to 1975 . However, what has been developed in the context of higher order continuum theories lacks the physical notions of surface strain, surface stress, and surface elastic moduli tensors which all are mathematically introduced by Gurtin and Murdoch (1975) in the frame- 
work of surface elasticity. More specifically, they treat the bulk material and its complementary surface as separate interacting entities, and write the equilibrium conditions of surfaces and interfaces in terms of the surface and interface stresses as well as bulk stresses. Along this line of thought, some researchers have additionally derived the driving force corresponding to the normal variation of a propagating interface (Gurtin, 1995; Simha and Bhattacharya, 1997, 1998, 2000). Moreover, Simha and Bhattacharya $(1997,1998,2000)$ have obtained the expressions for the driving force on the intersections of interfaces and where the interfaces meet the external boundary in terms of the bulk stress tensor, surface and interfacial stresses, bulk elastic energy, interfacial total energy, and surface energy. As it was alluded to, the other higher order continuum theories such as first and second strain gradient theories did not explicitly bring out the above-mentioned surface and interface parameters. Recently, Javili et al. (2013) consider the total energy of an elastic body as the sum of the energy of the bulk material and that of its bounding surface layer of finite thickness. In their work, the bulk and surface materials are assumed to be of grades 3 and 2, respectively. Moreover, in this context, at any field point within the surface layer, the first and second gradients of the displacement field in the thickness direction of the surface layer are nonzero. They carry out their formulations without reference to the constitutive relations associated with both bulk and its complementary surface. For this reason, the effect of certain physically important parameters such as modulus of cohesion and surface elastic constants in their analysis is ab- 
sent. Polizzotto (2013) considers an elastic body to consist of bulk and a surface layer with nonzero thickness. Polizzotto (2013) assumes that the bulk material is of grade 3 , but for simplification discards many of the corresponding terms from the energy expression; in particular, the term corresponding to the modulus of cohesion is missing from the analysis.

In first strain gradient theory, it is assumed that the potential energy density function of the material depends on both the second-order strain tensor and its first gradient (Mindlin and Eshel, 1968). The correspondence between first strain gradient theory and the atomic structure of the material is exhibited by Toupin and Gazis (1964) through consideration of the nearest and next nearest interatomic interactions; they realized that the drawing in or pushing out the surface layer happens only in non-centrosymmetric materials. Later, Toupin, in a private communication with Mindlin (1965), suggested that one can remove this restriction with the inclusion of the components of the second gradient of the strain tensor in the potential energy density function. Subsequently, Mindlin (1965) proposed second strain gradient theory in which the strain energy density function depends on, not only the strain field and its first derivative, but also the second derivative of the strain field. Agiasofitou and Lazar (2009) have explored the conservation and balance laws for second strain gradient elasticity employing Noether's theorem. In second strain gradient theory which is developed for centrosymmetric, homogeneous, and linearly elastic isotropic materials, sixteen elastic constants in addition to Lamé 
constants are revealed. Fifteen of these additional parameters bring on four characteristic lengths associated with the bulk and another length scale associated with the surface of such media. Generally speaking, bulk characteristic lengths together with Lamé constants appear in the equilibrium equations, while surface characteristic length enters the formulation through the boundary conditions, (Mindlin, 1965; Ojaghnezhad and Shodja, 2013). The remaining parameter is modulus of cohesion with the dimension of force which characterizes the surface effect. Mindlin (1965), when considering an elastic half-space with zero body force field and traction free surface within the framework of second strain gradient theory realized non-trivial solution due to the surface effect stemming from modulus of cohesion. Ojaghnezhad and Shodja (2013) by considering an infinitely extended traction free elastic film with zero body force field observed non-trivial solution and, moreover, derived an expression between the film's surface energy and modulus of cohesion.

As briefly mentioned earlier, Gurtin and Murdoch (1975), based on the prevailing capabilities of continuum mechanics, developed a mathematical framework for the mechanical behavior of elastic surfaces of materials. They bring about a concise notion of the material surface, surface strain tensor, surface stress, and surface elastic constants as well as the linearized theory of elastic surfaces. An interesting issue is the consideration of the simultaneous emergence of the two points of view, namely, surface elasticity theory of Gurtin and Murdoch (1975) and second strain gradient theory of Mindlin (1965). More specifically, one may ask whether there is 
a linkage between the surface elastic parameters such as surface stress and surface elastic constants of surface elasticity theory and the elastic parameters stemming from second strain gradient elasticity. To give an affirmative analysis to this concern, the current work attempts to revisit surface elasticity in the context of second strain gradient theory.

In the realm of experimentations, measurement of the additional parameters and characteristic lengths poses serious difficulties. On the other hand, to date, using a combination of simulation and analysis, such parameters associated with certain augmented continuum theories have been evaluated with some success. Shodja and Tehranchi $(2010,2012)$ and Shodja et al. (2012) calculated the values of the characteristic lengths of Mindlin's first and second strain gradient elasticity, respectively, for face-centered cubic (fcc) crystals using analytical formulations complemented by a many-body long-range Finnis-Sinclair potential (Finnis and Sinclair, 1984). In another contribution, ab-initio calculations based on density functional theory (DFT) are employed by Shodja et al. (2013) to calculate the additional parameters and characteristic lengths of Mindlin's first strain gradient elasticity. In the context of second strain gradient, Ojaghnezhad and Shodja (2013) gave an analysis combined with ab initio calculations to obtain modulus of cohesion, surface energy, and the additional constants. Moreover, Ojaghnezhad and Shodja (2012), in a separate study, calculated the surface elastic parameters such as surface residual stresses and surface elastic constants of the ideal and reconstructed surfaces of $\mathrm{Si}(001)$ using 
first principles computations. In the present work, however, for a film of infinite extension, the surface elastic parameters which appear in surface elasticity such as surface residual stresses, surface energy, and surface elastic constants are analytically related to Mindlin's additional parameters introduced in second strain gradient theory. Afterwards, by utilizing the available experimental values of surface energy and surface residual stresses, the corresponding relations are solved simultaneously for surface characteristic length and modulus of cohesion. To this end, the values of Lamé constants and bulk characteristic lengths must be known as well; these parameters will be computed using ab initio DFT calculations. In continue, surface elastic constants and an estimate of the effective elastic constants for each considered film are determined.

This paper proceeds as below. Section 2, in the realm of surface elasticity theory, recovers some preliminary definitions for surface gradient and surface divergence theorem. In section 3, the theory of surface elasticity is developed in the framework of second strain gradient elasticity with energy considerations included. Section 4 is devoted to a film of infinite extension with planar surfaces in an effort to give analytical expressions for the residual surface stress tensor and surface elastic constants, formulate the effective elastic constants, and lay down a methodology for the calculation of surface characteristic length and modulus of cohesion, all in the mathematical framework of second strain gradient theory. In section 5 , by utilizing theory of lattice dynamics, a treatment for obtaining Lamé constants and bulk char- 
acteristic lengths in terms of atomic force constants is developed. In Section 6, case studies are performed on three fcc crystals, Ag, Au, and Pt. For these materials, the surface relaxations calculated using the current theory are compared with those obtained via TB (Rosato et al., 1989) as well as experiment (Kuk and Feldman, 1984).

\section{Preliminary definitions}

An immediate purpose of the current work is to develop surface elasticity theory in the context of second strain gradient elasticity. Thus, to the aim of selfcontentedness of the paper, a brief presentation of the required definitions and results previously given by Gurtin and Murdoch (1975) in some detail is in order. Let $\mathscr{E}$ be a three-dimensional Euclidean point space and $\mathscr{V}$ denote the associated translation space. Consider a three-dimensional elastic body $\mathscr{B}$ with the elastic material surface $\mathscr{S}=\partial \mathscr{B}$. Assume that the region $B \in \mathscr{E}$ is the image of $\mathscr{B}$ in a fixed reference configuration and $s=\partial B$, which is the image of $\mathscr{S}$, is a smooth orientable surface with outward unit normal field, $\boldsymbol{n}$. Assume that $\mathscr{T}_{\boldsymbol{x}}$ is a two-dimensional subspace of $\mathscr{V}$ and denotes the tangent space at $\boldsymbol{x} \in \boldsymbol{s}$. Between the three-dimensional space, $\mathscr{V}$ and its two-dimensional subspace, $\mathscr{T}_{\boldsymbol{x}}$, Gurtin and Murdoch (1975) define the inclusion map $\mathbf{I}(\boldsymbol{x})$ as a linear transformation from $\mathscr{T}_{\boldsymbol{x}}$ to $\mathscr{V}$, displayed as $\mathbf{I}(\boldsymbol{x}) \in \operatorname{Lin}\left(\mathscr{T}_{\boldsymbol{x}}, \mathscr{V}\right)$, with the property $\mathbf{I}(\boldsymbol{x}) \cdot \boldsymbol{\tau}=\boldsymbol{\tau}$ for every $\boldsymbol{\tau} \in \mathscr{T}_{\boldsymbol{x}}$. Conversely,

the perpendicular projection, $\boldsymbol{P}(\boldsymbol{x}) \in \operatorname{Lin}\left(\mathscr{V}, \mathscr{T}_{\boldsymbol{x}}\right)$ is defined as the transpose of $\mathbf{I}(\boldsymbol{x})$ 
and hence $\boldsymbol{P}(\boldsymbol{x}) \cdot \mathbf{I}(\boldsymbol{x})$ gives the identity map on $\mathscr{T}_{\boldsymbol{x}}$ which is denoted by $1(\boldsymbol{x})$. Due to the above definitions, $\mathbf{I}(\boldsymbol{x}) \cdot \boldsymbol{P}(\boldsymbol{x}) \in \operatorname{Lin}(\mathscr{V}, \mathscr{V})$ and

$$
\mathbf{I}(\boldsymbol{x}) \cdot \boldsymbol{P}(\boldsymbol{x})=\mathbf{1}-\boldsymbol{n}(\boldsymbol{x}) \otimes \boldsymbol{n}(\boldsymbol{x})
$$

where $\mathbf{1}$ is the identity map on $\mathscr{V}$. For clarity, some of the formulations in the subsequent analysis are presented using indicial notation with summation convention. Moreover, throughout this paper, the rules

$$
\boldsymbol{a b}: \boldsymbol{c d}=(\boldsymbol{a} . \boldsymbol{c})(\boldsymbol{b} . \boldsymbol{d}), \quad \boldsymbol{a b c}: \boldsymbol{d e f}=(\boldsymbol{a} . \boldsymbol{d})(\boldsymbol{b} . \boldsymbol{e})(\boldsymbol{c} . \boldsymbol{f}), \quad \text { etc. }
$$

respectively, are used for scalar multiplication of dyads, triads, etc. The operator for gradient on $s$ is defined as (Gurtin and Murdoch, 1975)

$$
\nabla_{s}=P \cdot \nabla
$$

It is readily shown that $\mathbf{I} \cdot \nabla_{\jmath}=\boldsymbol{\nabla}-\boldsymbol{n n} . \boldsymbol{\nabla}$ where $\boldsymbol{\nabla}$ is the gradient on $B$. For an arbitrary smooth vector field, $\boldsymbol{v}$ on $s$ defined as $\boldsymbol{v}: \mathfrak{s} \rightarrow \mathscr{V}$, the components of $\boldsymbol{\nabla}_{\jmath} \boldsymbol{v} \in \operatorname{Lin}\left(\mathscr{V}, \mathscr{T}_{\boldsymbol{x}}\right)$ are defined as

$$
\nabla_{\jmath} v=P \cdot \nabla v
$$

The tangential derivative of $\boldsymbol{v}$ which belongs to $\operatorname{Lin}\left(\mathscr{T}_{\boldsymbol{x}}, \mathscr{T}_{\boldsymbol{x}}\right)$ is $\left(\boldsymbol{\nabla}_{\boldsymbol{s}} \boldsymbol{v}\right) . \mathbf{I}$ and the surface divergence is defined as

$$
\operatorname{div}_{\jmath} \boldsymbol{v}=\operatorname{tr}\left(\left(\boldsymbol{\nabla}_{\jmath} \boldsymbol{v}\right) . \mathbf{I}\right)
$$

From calculus, it is known that for a continuously differentiable tensor point function, $\boldsymbol{f}$ of any order over an orientable regular subsurface, $\Sigma \subset s$ with unit 
normal, $\boldsymbol{n}$ and piecewise smooth boundary curve, $\partial \Sigma$ with positive unit tangent $\mathrm{T}$, the following relation holds (Brand, 1966)

$$
\int_{\Sigma}\left(\boldsymbol{n}^{2} \cdot \nabla \boldsymbol{f}-\boldsymbol{n} \nabla \cdot \boldsymbol{f}\right)=\oint_{\partial \Sigma} \mathrm{T} \times \boldsymbol{f}
$$

where the number 2 over the dot denotes that $\boldsymbol{n}$ is dotted into the second vector to the right of the dot. By replacing $\boldsymbol{f}$ with $\boldsymbol{n} \boldsymbol{f}$ in the left hand side of Eq. (2.6), we have

$$
\int_{\Sigma}\left(\boldsymbol{n}^{2} \cdot \nabla(\boldsymbol{n} \boldsymbol{f})-\boldsymbol{n} \nabla \cdot(\boldsymbol{n} \boldsymbol{f})\right)=\int_{\Sigma}\left(\mathbf{I} \cdot \nabla_{\lrcorner}-\left(\mathbf{I} \cdot \nabla_{\jmath} \cdot \boldsymbol{n}\right) \boldsymbol{n}\right) \boldsymbol{f}
$$

where $\boldsymbol{n} \boldsymbol{\nabla} \boldsymbol{n}=\mathbf{0}$ has been used. Subsequently, the following identity is obtained

$$
\int_{\Sigma} \boldsymbol{L} \cdot \boldsymbol{f}=\int_{\partial \Sigma} \boldsymbol{m} \cdot \boldsymbol{f}
$$

with

$$
\boldsymbol{L}=\mathbf{I} . \nabla_{\jmath}-\left(\mathbf{I} \cdot \nabla_{\jmath} . n\right) n
$$

and $\boldsymbol{m}=\mathrm{T} \times \boldsymbol{n}$ which is the outward unit normal to $\partial \Sigma$ at $\boldsymbol{x}$ and $\boldsymbol{m}(\boldsymbol{x}) \in \operatorname{Unit}\left(\mathscr{T}_{\boldsymbol{x}}\right)$; $\operatorname{Unit}\left(\mathscr{T}_{\boldsymbol{x}}\right)$ is the set of all unit vectors in $\mathscr{T}_{\boldsymbol{x}}$. Subsequently, for a smooth tangent vector field, $\boldsymbol{t}$ on $\Sigma$,

$$
\int_{\Sigma} \operatorname{div}_{\jmath} t=\int_{\partial \Sigma} \boldsymbol{m} . \boldsymbol{t} .
$$

In the case of regular geometrically closed surface, $\int_{\Sigma} \operatorname{div}_{\jmath} \boldsymbol{t}=0$. Also, for a smooth

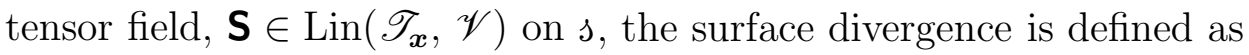

$$
\operatorname{div}_{\jmath} \mathbf{S}=\mathbf{I}: \nabla \mathbf{S}^{T}
$$


and associated with $\Sigma \subset$ s, the surface divergence theorem is

$$
\int_{\partial \Sigma} \mathbf{S} \cdot \boldsymbol{m}=\int_{\Sigma} \operatorname{div}_{\jmath} \mathbf{S}
$$

\section{Reformulation of surface elasticity in the context of second strain gra- dient theory}

Previously, Ojaghnezhad and Shodja (2013) have calculated modulus of cohesion and its relation to the surface energy associated with a film in the context of second strain gradient theory only. Throughout this section, surface elasticity theory of Gurtin and Murdoch (1975) will be reformulated in the mathematical framework of second strain gradient theory of Mindlin (1965) in an effort to evaluate surface stresses, surface elastic constants, and effective elastic constants associated with a film within the present developments. To this end, in the kinematical considerations, in addition to the bulk second order strain tensor and its first and second derivatives, their corresponding complementary surface tangential tensors are defined and in continue the equilibrium equations for both bulk and surface are written.

\subsection{Kinematics}

Suppose that an elastic medium $\mathscr{B}$ with an enclosing surface $\partial \mathscr{B}$ occupies the region $B$ with respect to a fixed reference configuration. According to the linear second strain gradient theory formulated by Mindlin (1965), in addition to the usual

second order strain tensor, $\stackrel{\boldsymbol{\epsilon}}{\boldsymbol{\epsilon}}$ the first and second gradients of strains are also taken 
into consideration via $\stackrel{2}{\boldsymbol{\epsilon}}$ and $\stackrel{3}{\boldsymbol{\epsilon}}$ as defined below

$$
\begin{aligned}
& \stackrel{1}{\boldsymbol{\epsilon}}=\frac{1}{2}(\nabla \boldsymbol{u}+\boldsymbol{u} \nabla), \\
& \stackrel{2}{\boldsymbol{\epsilon}}=\nabla \nabla \boldsymbol{u}, \\
& \stackrel{3}{\boldsymbol{\epsilon}}=\nabla \nabla \nabla \boldsymbol{u},
\end{aligned}
$$

where $\boldsymbol{u}: B \rightarrow \mathscr{V}$ is the displacement vector. For the points on the material surface, define the displacement vector $\boldsymbol{u}: \partial B \rightarrow \mathscr{V}$. The infinitesimal surface strain tensor is considered as the tangential field (Gurtin and Murdoch, 1975)

$$
\stackrel{1}{\mathbf{E}}=\frac{1}{2}\left(\boldsymbol{P} \cdot \boldsymbol{u} \overleftarrow{\nabla}_{\jmath}+\vec{\nabla}_{\jmath} \boldsymbol{u} . \mathbf{I}\right)
$$

For further enhancement, one may also consider the first and second surface gradients of the surface strain tensor as

$$
\begin{aligned}
& \stackrel{2}{\mathbf{E}}=\vec{\nabla}_{\jmath} \vec{\nabla}_{\jmath} \boldsymbol{u} . \mathbf{I}, \\
& \stackrel{3}{\mathbf{E}}=\vec{\nabla}_{\jmath} \vec{\nabla}_{\jmath} \vec{\nabla}_{\jmath} \boldsymbol{u} . \mathbf{I} .
\end{aligned}
$$

In the remaining analysis, the strain tensor, $\stackrel{\boldsymbol{\epsilon}}{\boldsymbol{\epsilon}}$ and its first and second gradients, $\stackrel{2}{\boldsymbol{\epsilon}}$ and $\stackrel{3}{\boldsymbol{\epsilon}}$ will be kept but for the sake of simplicity it is assumed that the second order tensor, $\mathbf{E}=\mathbf{E}$ is a sufficiently accurate measure of the tangential surface deformation.

\subsection{Equilibrium equations in the bulk and boundary conditions}

According to Mindlin (1965), the variation of the total elastic strain energy in a volume $B$ with the material boundary $\partial B$, in the absence of any edges and corners 
is

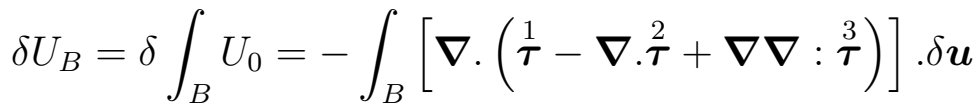

$$
\begin{aligned}
& +\int_{\partial B} \boldsymbol{n} \cdot\left(\stackrel{1}{\boldsymbol{\tau}}-\boldsymbol{\nabla} \cdot \stackrel{2}{\boldsymbol{\tau}}^{2} \boldsymbol{\nabla} \boldsymbol{\nabla}: \stackrel{3}{\boldsymbol{\tau}}\right) \cdot \delta \boldsymbol{u} \\
& -\int_{\partial B}\left[\boldsymbol{L} \cdot\left(\boldsymbol{n} \cdot\left(\stackrel{2}{\boldsymbol{\tau}}-\nabla \cdot \dot{3}^{\boldsymbol{\tau}}\right)-\boldsymbol{L} \cdot\left(\boldsymbol{n} \cdot \boldsymbol{3}^{\boldsymbol{\tau}}\right)-\left(\mathbf{I} \cdot \nabla_{\jmath} \boldsymbol{n}\right) \cdot(\boldsymbol{n} \boldsymbol{n}: \stackrel{3}{\boldsymbol{\tau}})\right)\right] . \delta \boldsymbol{u} \\
& -\int_{\partial B}\left(\boldsymbol{n} \cdot \boldsymbol{L} \cdot\left(\boldsymbol{n} \cdot \stackrel{3}{\boldsymbol{\tau}}^{2}\right)+\boldsymbol{L} \cdot\left(\boldsymbol{n n}: \stackrel{3}{\boldsymbol{\tau}}^{2}-\boldsymbol{n n}:\left(\stackrel{2}{\boldsymbol{\tau}}-\nabla \cdot \frac{3}{\boldsymbol{\tau}}\right)\right) \cdot D \delta \boldsymbol{u}\right. \\
& +\int_{\partial B}\left(\boldsymbol{n n n}: \frac{3}{\tau}\right) \cdot D^{2} \delta \boldsymbol{u}
\end{aligned}
$$

where $U_{0}$ is the elastic strain energy density and

$$
\begin{aligned}
& \stackrel{1}{\boldsymbol{\tau}}=\frac{\partial U_{0}}{\partial \boldsymbol{\epsilon}}, \\
& \stackrel{2}{\boldsymbol{\tau}}=\frac{\partial U_{0}}{\partial^{2} \boldsymbol{\epsilon}}, \\
& \stackrel{3}{\boldsymbol{\tau}}=\frac{\partial U_{0}}{\partial^{3} \boldsymbol{\epsilon}},
\end{aligned}
$$

are the generalized stress tensors. The operator $D$ in Eq. (3.4) is defined as $D=\boldsymbol{n} . \boldsymbol{\nabla}$ and $\boldsymbol{L}$ is given by Eq. (2.9). The virtual work, $\delta W_{\text {virt }}$ of the external applied loads is written in the following form

$$
\delta W_{\mathrm{virt}}=\int_{B} \boldsymbol{b}_{B} \cdot \delta \boldsymbol{u}+\int_{\partial B}\left(\stackrel{1}{\boldsymbol{t}} . \delta \boldsymbol{u}+\stackrel{2}{\boldsymbol{t}} \cdot D \delta \boldsymbol{u}+\stackrel{3}{\boldsymbol{t}} \cdot D^{2} \delta \boldsymbol{u}\right)
$$

where $\boldsymbol{b}_{B}$ is the body force of the volume and $\stackrel{1}{\boldsymbol{t}}, \stackrel{2}{\boldsymbol{t}}$, and $\stackrel{3}{\boldsymbol{t}}$ are the surface tractions applied on the material boundary. The principle of stationary potential energy requires that $\delta\left(U_{B}-W_{\text {virt }}\right)=0$, which subsequently leads to

$$
\boldsymbol{\nabla} . \boldsymbol{S}+\boldsymbol{b}_{B}=\mathbf{0},
$$


in $B$, and the generalized surface tractions

$$
\begin{aligned}
& \stackrel{1}{\boldsymbol{t}}=\boldsymbol{n} \cdot \boldsymbol{S}-\boldsymbol{L} \cdot \stackrel{\boldsymbol{s}}{\boldsymbol{\tau}} \\
& \stackrel{2}{\boldsymbol{t}}=\boldsymbol{n n}:\left(2^{\boldsymbol{\tau}}-\nabla \cdot \frac{3}{\boldsymbol{\tau}}\right)-\boldsymbol{n} \cdot(\boldsymbol{L} \cdot(\boldsymbol{n} \cdot \stackrel{3}{\boldsymbol{\tau}}))-\boldsymbol{L} \cdot(\boldsymbol{n n}: \stackrel{3}{\boldsymbol{\tau}}) \\
& \stackrel{3}{\boldsymbol{t}}=\boldsymbol{n n n}: \stackrel{3}{\boldsymbol{\tau}}
\end{aligned}
$$

on $\partial B$, where

$$
\begin{aligned}
& \boldsymbol{S}=\stackrel{1}{\boldsymbol{\tau}}-\nabla \cdot \stackrel{2}{\boldsymbol{\tau}}+\nabla \nabla: \stackrel{3}{\boldsymbol{\tau}} \\
& \stackrel{\boldsymbol{s}}{\boldsymbol{\tau}}=\boldsymbol{n} \cdot(\stackrel{2}{\boldsymbol{\tau}}-\nabla \cdot \stackrel{3}{\boldsymbol{\tau}})-\boldsymbol{L} \cdot(\boldsymbol{n} \cdot \stackrel{3}{\boldsymbol{\tau}})-\left(\mathbf{I} \cdot \nabla_{\jmath} \boldsymbol{n}\right) \cdot(\boldsymbol{n n}: \stackrel{3}{\boldsymbol{\tau}})
\end{aligned}
$$

Assume that $\partial B$ is a regular closed surface, then by letting $\Sigma \equiv \partial B$ and $\boldsymbol{f} \equiv \stackrel{\boldsymbol{s}}{\boldsymbol{\tau}}$ in Eq. (2.8), it results in

$$
\int_{\partial B} \boldsymbol{L} \cdot \boldsymbol{s}=0
$$

Upon integration of the equilibrium equation over the region $B$, and employment of the divergence theorem to the first part leads to

$$
\int_{\partial B} \boldsymbol{n} . \boldsymbol{S}+\int_{B} \boldsymbol{b}_{B}=\mathbf{0 .}
$$

With due attention to Eqs. (3.8a) and (3.10), Eq. (3.11) reduces to

$$
\int_{B} \boldsymbol{b}_{B}+\int_{\partial B} \stackrel{1}{\boldsymbol{t}}=\mathbf{0}
$$

implying that the total body force of the bulk is in equilibrium with the total traction of the first kind. 
According to Mindlin (1965), the strain energy density for homogeneous, isotropic, and centrosymmetric materials has the following form

$$
\begin{aligned}
U_{0} & =\frac{1}{2} \lambda \epsilon_{i i} \epsilon_{j j}+\mu \epsilon_{i j} \epsilon_{i j}+a_{1} \epsilon_{i j j} \epsilon_{i k k}+a_{2} \epsilon_{i i k} \epsilon_{k j j}+a_{3} \epsilon_{i i k} \epsilon_{j j k}+a_{4} \epsilon_{i j k} \epsilon_{i j k} \\
& +a_{5} \epsilon_{i j k} \epsilon_{k j i}+b_{1} \epsilon_{i i j j} \epsilon_{k k l l}+b_{2} \epsilon_{i j k k} \epsilon_{i j l l}+b_{3} \epsilon_{i i j k} \epsilon_{j k l l}+b_{4} \epsilon_{i i j k} \epsilon_{l l k j} \\
& +b_{5} \epsilon_{i i j k} \epsilon_{l l j k}+b_{6} \epsilon_{i j k l} \epsilon_{i j k l}+b_{7} \epsilon_{i j k l} \epsilon_{j k l i}+c_{1} \epsilon_{i i} \epsilon_{j j k k}+c_{2} \epsilon_{i j} \epsilon_{i j k k} \\
& +c_{3} \epsilon_{i j} \epsilon_{k k i j}+b_{0} \epsilon_{i i j j}
\end{aligned}
$$

where $\epsilon_{i j}, \epsilon_{i j k}$, and $\epsilon_{i j k l}$ are the components of $\stackrel{1}{\boldsymbol{\epsilon}}, \stackrel{2}{\boldsymbol{\epsilon}}$, and $\stackrel{3}{\boldsymbol{\epsilon}}$, respectively. $\lambda$ and $\mu$ are Lamé constants and $a_{i}$ 's, $b_{i}$ 's, and $c_{i}$ 's are the additional constants corresponding to Mindlin's second strain gradient theory. The bulk characteristic lengths, $\ell_{11}, \ell_{12}$, $\ell_{21}$, and $\ell_{22}$ in Mindlin's second strain gradient theory are defined as

$$
\begin{aligned}
& 2(\lambda+2 \mu) \ell_{1 j}^{2}=\bar{a}-2 \bar{c} \pm\left\{(\bar{a}-2 \bar{c})^{2}-4 \bar{b}(\lambda+2 \mu)\right\}^{1 / 2} \\
& 2 \mu \ell_{2 j}^{2}=\bar{a}^{\prime}-c_{3} \pm\left\{\left(\bar{a}^{\prime}-c_{3}\right)^{2}-4 \bar{b}^{\prime} \mu\right\}^{1 / 2}
\end{aligned}
$$

where $j=1$ and 2 correspond to the plus and minus signs, respectively and

$$
\begin{aligned}
& \bar{a}=2\left(a_{1}+a_{2}+a_{3}+a_{4}+a_{5}\right), \\
& \bar{b}=2\left(b_{1}+b_{2}+b_{3}+b_{4}+b_{5}+b_{6}+b_{7}\right), \\
& \bar{c}=c_{1}+c_{2}+c_{3}, \\
& \bar{a}^{\prime}=2\left(a_{3}+a_{4}\right), \\
& \bar{b}^{\prime}=2\left(b_{5}+b_{6}\right) .
\end{aligned}
$$




\subsection{The relation between Piola-Kirchhoff surface stress and bulk stress tensors}

As it was alluded to, the bulk material obeys second strain gradient theory and thus it is of interest to express the net Piola-Kirchhoff surface stress tensor on $\partial B$ in terms of $\stackrel{1}{\boldsymbol{\tau}}, \stackrel{2}{\boldsymbol{\tau}}, \stackrel{3}{\boldsymbol{\tau}}, \nabla_{\boldsymbol{s}} \boldsymbol{n}$, and $\boldsymbol{n}$ within $B$. Gurtin and Murdoch (1975) have shown that if the surface traction field $\boldsymbol{t}_{\mathfrak{s}}$ on $\boldsymbol{s}$ and the surface body force field $\boldsymbol{b}_{\mathfrak{s}}(\boldsymbol{x})$, which represents the force per unit area exerted on $\boldsymbol{s}$ at $\boldsymbol{x}$ by the environment, are in equilibrium, then the following relation holds

$$
\operatorname{div}_{\jmath} \mathbf{S}+\boldsymbol{b}_{\jmath}=\mathbf{0}, \quad \text { on } \quad \partial B
$$

where $\mathbf{S}(\boldsymbol{x}) \in \operatorname{Lin}\left(\mathscr{T}_{\boldsymbol{x}}, \mathscr{V}\right)$ is the Piola-Kirchhoff surface stress tensor on $\mathfrak{s}$. Note that the Piola-Kirchhoff surface stress tensor is not symmetric. As explained in the previous section, the force per unit undeformed area $-\stackrel{1}{\boldsymbol{t}}$ which is determined via Eq. (3.8a), is exerted on $\partial B$ by the interior of $B$, which here we denote it as $\stackrel{\circ}{B}$. Then, the total body force on the surface $s$ is given as

$$
\boldsymbol{b}_{\lrcorner}=\boldsymbol{b}_{e}-\stackrel{1}{\boldsymbol{t}}
$$

where $\boldsymbol{b}_{e}$ includes any force per unit area other than $\stackrel{-1}{-\boldsymbol{t}}$ which is applied on the surface $s$ by the environment. Integrating Eq. (3.16) over $s=\partial B$ and using Eqs. (2.12), (3.17), and (3.12) result in

$$
\int_{\partial(\partial B)} \mathbf{S} \cdot \boldsymbol{m}+\int_{B} \boldsymbol{b}_{B}+\int_{\partial B} \boldsymbol{b}_{e}=\mathbf{0}
$$

For regular closed surfaces $\int_{\partial(\partial B)} \mathbf{S} \cdot \boldsymbol{m}=\mathbf{0}$, and hence

$$
\int_{B} \boldsymbol{b}_{B}+\int_{\partial B} \boldsymbol{b}_{e}=0
$$


In accordance to the linearized theorem given by Gurtin and Murdoch (1975), the Piola-Kirchhoff surface stress tensor is obtained as

$$
\mathbf{S}=\mathbf{I} \cdot \mathbf{M}+\mathbf{I} \cdot \mathbf{C}: \mathbf{E}+\left(\boldsymbol{u} \overleftarrow{\nabla}_{s}\right) \cdot \mathbf{M}
$$

where the symmetric tangential second order tensor, $\mathbf{M}$ is the residual surface stress at $\boldsymbol{x}$ in the undeformed configuration and $\mathbf{C}$ is the surface elasticity tensor which transforms the symmetric tangential strain tensor, $\mathbf{E}$ into a symmetric tangential tensor, $\mathbf{C}$ : $\mathbf{E}$. Due to the symmetries of $\mathbf{E}$ and $\mathbf{C}: \mathbf{E}$, and assuming the hyperelasticity properties as for the bulk, $\mathbf{C}$ has six independent components. In $x_{2} x_{3}$-plane, the independent components of $\mathbf{C}$ are $\mathrm{C}_{2222}, \mathrm{C}_{2223}, \mathrm{C}_{2233}, \mathrm{C}_{2323}, \mathrm{C}_{2333}$, and $\mathrm{C}_{3333}$. Using the standard theorem for linear, isotropic and symmetric tensor-valued functions of a symmetric tensor variable (Gurtin and Murdoch, 1975), it is inferred that

$$
\begin{aligned}
& \mathbf{M}=\sigma_{0} 1, \\
& \mathbf{C}: \mathbf{E}=\lambda_{0}(\operatorname{tr} \mathbf{E}) 1+2 \mu_{0} \mathbf{E},
\end{aligned}
$$

in which $\sigma_{0}, \lambda_{0}$, and $\mu_{0}$ depend on the material point $\boldsymbol{x}$. It is noteworthy to mention that the tangential tensor, $\mathbf{M}$ represented by Eq. (3.21a) is referred to as surface tension. Define the tensor of curvature for surface $s$ in the undeformed configuration as

$$
\kappa=-P \cdot\left(n \overleftarrow{\nabla}_{s}\right)
$$

Noting that $\boldsymbol{\kappa} \cdot \boldsymbol{P}=-\vec{\nabla}_{\jmath} \boldsymbol{n}$, and the tangential tensors $\mathbf{M}, \mathbf{C}: \mathbf{E}$, and $\boldsymbol{\kappa}$ are symmet- 


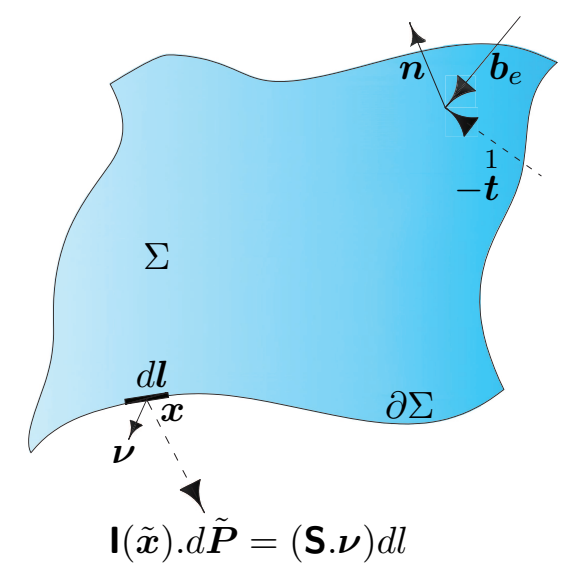

a. undeformed configuration

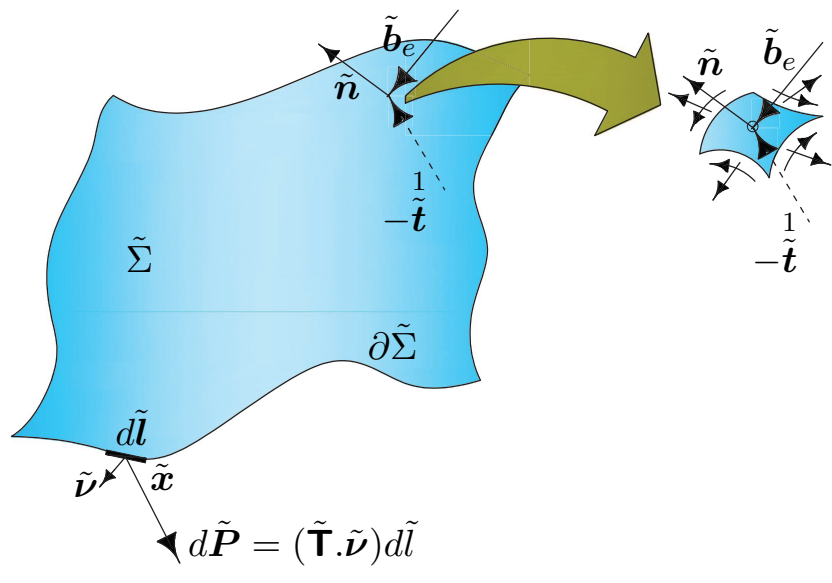

b. deformed configuration

Figure 1: (a) $\Sigma$ is any regular subsurface of the smooth orientable surface $s$ associated with the undeformed configuration and is bounded by an oriented smooth curve $\partial \Sigma$. $\boldsymbol{b}_{e}$ is the current body force per unit undeformed area and $-\stackrel{t}{t}$ is the current force per unit undeformed area exerted by the bulk. $d \tilde{\boldsymbol{P}}$ is the actual force in the deformed configuration. $d \boldsymbol{l}$ is an oriented line element at $\boldsymbol{x}$ along $\partial \Sigma . \boldsymbol{\nu}$ is a unit vector lying in the plane tangent to $\Sigma$ at the point $\boldsymbol{x}$; (b) deformed configuration and its associated field quantities $\tilde{\boldsymbol{b}}_{e},-\tilde{\boldsymbol{\boldsymbol { t }}}$, and $d \tilde{\boldsymbol{P}}$. In this configuration not only $\boldsymbol{\nu}$ but also $d \tilde{\boldsymbol{P}}$ lies in the plane tangent to $\tilde{\Sigma}$ at the point $\tilde{\boldsymbol{x}}$. The equilibrium of the surface stresses with $\tilde{\boldsymbol{b}}_{e}$ and $-\tilde{\boldsymbol{t}}$ acting on a surface element with unit outward normal $\boldsymbol{n}$ is demonstrated in the magnified element on the right.

ric, then by employing the identity

$$
\left(\operatorname{div}_{s} \mathbf{S}\right) \cdot \boldsymbol{n}=\operatorname{div}_{\jmath}\left(\mathbf{S}^{T} \cdot \boldsymbol{n}\right)-\mathbf{S}: \boldsymbol{n} \overleftarrow{\nabla}_{\jmath}
$$

and Eq. (3.20), it is deduced that

$$
\left(\operatorname{div}_{\jmath} \mathbf{S}\right) \cdot \boldsymbol{n}=\operatorname{div}_{\jmath}\left(\mathbf{S}^{T} \cdot \boldsymbol{n}\right)+\boldsymbol{\kappa}:\left(\mathbf{M}+\mathbf{C}: \mathbf{E}+\boldsymbol{P} \cdot\left(\boldsymbol{u} \overleftarrow{\boldsymbol{\nabla}}_{\jmath}\right) \cdot \mathbf{M}\right) .
$$

With the aid of Eqs. (3.16), (3.17), and (3.20), it can be shown that

$$
\operatorname{div}_{\jmath}\left(\mathbf{M} \cdot\left(\vec{\nabla}_{\jmath} \boldsymbol{u}\right) \cdot \boldsymbol{n}\right)+\boldsymbol{\kappa}:(\boldsymbol{P} . \mathbf{S})=\boldsymbol{n} \cdot\left(\boldsymbol{t}-\boldsymbol{b}_{e}\right) .
$$

It may be emphasized that the surface Cauchy stress tensor, $\tilde{\mathbf{T}}$ on any regular subsurface, $\tilde{\Sigma}$ of $\tilde{\jmath}$ associated with the deformed configuration is a tangential tensor 
and hence, as schematically illustrated in Fig. 1b, the force, $d \tilde{\boldsymbol{P}}$ acting on the line element $d \tilde{\boldsymbol{l}}$ at $\tilde{\boldsymbol{x}}$ along the smooth curve $\partial \tilde{\Sigma}$ of the deformed surface is tangent to $\tilde{\Sigma}$ at $\tilde{\boldsymbol{x}}$. The Piola-Kirchhoff surface stress tensor, S, however, is the surface stress tensor such that $(\mathbf{S} . \boldsymbol{\nu}) d l=\mathbf{I}(\tilde{\boldsymbol{x}}) \cdot d \tilde{\boldsymbol{P}}=d \tilde{\boldsymbol{P}}=(\tilde{\mathbf{T}} \cdot \tilde{\boldsymbol{\nu}}) d \tilde{l}$, where the unit outward normals $\boldsymbol{\nu}$ and $\tilde{\boldsymbol{\nu}}$ to the oriented line elements $d \boldsymbol{l}$ and $d \tilde{\boldsymbol{l}}$, respectively, lie in the tangent planes to $\Sigma$ and $\tilde{\Sigma}$ at $\boldsymbol{x}$ and $\tilde{\boldsymbol{x}}$. Thus, in general, the actual force $d \tilde{\boldsymbol{P}}$ is not necessarily tangent to the undeformed surface, $\Sigma$ at the corresponding point $\boldsymbol{x}$. In the special case where associated to any point on every oriented curve in $\Sigma, \mathbf{S} . \boldsymbol{\nu}$ is a vector tangent to the undeformed surface, $\Sigma$ at that point, one may represent $\mathbf{S}=\mathbf{I}(\boldsymbol{x}) . \mathbf{T}$, where $\mathbf{T}$ is a tangential tensor. Thus, $\boldsymbol{n} . \mathbf{S}=\boldsymbol{n . I}(\boldsymbol{x}) . \mathbf{T}=\mathbf{0}$ since, $\boldsymbol{P} . \boldsymbol{n}=$ 0. Subsequently, the first term on the right hand side of Eq. (3.23) and the first term on the left hand side of Eq. (3.25) vanish, regardless of the curvature of 3. Thus, under this condition and such a scenario, where the curvature tensor is zero, $\boldsymbol{n} .\left(\stackrel{1}{\boldsymbol{t}}-\boldsymbol{b}_{e}\right)=0$; moreover, for free surfaces, $\boldsymbol{b}_{e}=\mathbf{0}$ and so $\boldsymbol{n} . \boldsymbol{t}=0$.

Now, dyade both sides of Eq. (3.7) with an arbitrary vector $\boldsymbol{v} \in \mathscr{V}$ and integrate over the region $B$

$$
\int_{B}(\operatorname{div} \boldsymbol{S}) \otimes \boldsymbol{v}=-\int_{B} \boldsymbol{b}_{B} \otimes \boldsymbol{v}
$$

By employing the divergence theorem

$$
\int_{\partial B} \boldsymbol{n} \cdot(\boldsymbol{S} \otimes \boldsymbol{v})-\int_{B} \boldsymbol{S}^{T}(\boldsymbol{\nabla} \otimes \boldsymbol{v})=-\int_{B} \boldsymbol{b}_{B} \otimes \boldsymbol{v} .
$$

Assuming that $\boldsymbol{v}$ is the position vector, $\boldsymbol{x}$ and using $\boldsymbol{\nabla} \boldsymbol{x}=\mathbf{1}$ then Eq. (3.27) reduces 
to

$$
\int_{\partial B} \boldsymbol{n} . \boldsymbol{S} \otimes \boldsymbol{x}=\int_{B} \boldsymbol{S}^{T}-\int_{B} \boldsymbol{b}_{B} \otimes \boldsymbol{x} .
$$

Employing Eqs. (3.8a), (3.16), and (3.17), it is seen that

$$
\int_{B} \boldsymbol{S}^{T}=\int_{\partial B}\left(\operatorname{div}_{\jmath} \mathbf{S}+\boldsymbol{L} \cdot \stackrel{s}{\boldsymbol{\tau}}\right) \otimes \boldsymbol{x}+\int_{B} \boldsymbol{b}_{B} \otimes \boldsymbol{x}+\int_{\partial B} \boldsymbol{b}_{e} \otimes \boldsymbol{x}
$$

By using the identity

$$
\int_{\partial \Sigma} \mathbf{S} \cdot \boldsymbol{m} \otimes \boldsymbol{x}=\int_{\Sigma}\left(\left(\operatorname{div}_{\jmath} \mathbf{S}\right) \otimes \boldsymbol{x}+\mathbf{S} . \nabla_{\jmath} \boldsymbol{x}\right)
$$

for a regular closed surface $\partial B$, the first term on the right-hand side of Eq. (3.29) may be written as

$$
\int_{\partial B}\left(\operatorname{div}_{\jmath} \mathbf{S}\right) \otimes \boldsymbol{x}=-\int_{\partial B} \mathbf{S} . \boldsymbol{P} .
$$

In arriving at Eq. (3.31), the Eq. (2.4) has also been utilized. To simplify the second term on the right hand side of Eq. (3.29), consider a second order tensor $\boldsymbol{f}$ and a vector $\boldsymbol{v} \in \mathscr{V}$ on an orientable surface $\Sigma$ with unit outward normal $\boldsymbol{n}$ and boundary $\partial \Sigma$. Then

$$
\begin{aligned}
\int_{\Sigma}\left(\boldsymbol{n}^{2} \cdot \nabla \boldsymbol{f}-\boldsymbol{n} \boldsymbol{\nabla} \cdot \boldsymbol{f}\right) \otimes \boldsymbol{v} & =\int_{\Sigma}\left(\boldsymbol{n}^{2} \cdot \nabla(\boldsymbol{f} \otimes \boldsymbol{v})-\boldsymbol{n} \nabla \cdot(\boldsymbol{f} \otimes \boldsymbol{v})\right) \\
& +\int_{\Sigma} n_{i} f_{j l} \frac{\partial v_{m}}{\partial x_{j}} \boldsymbol{e}_{i} \boldsymbol{e}_{l} \boldsymbol{e}_{m}-\int_{\Sigma} n_{i} f_{i l} \frac{\partial v_{m}}{\partial x_{j}} \boldsymbol{e}_{j} \boldsymbol{e}_{l} \boldsymbol{e}_{m}
\end{aligned}
$$

where $\boldsymbol{e}_{i}$ is the unit base vector. Employing the identity (2.6)

$$
\begin{aligned}
\int_{\Sigma}\left(\boldsymbol{n}^{2} \cdot \nabla \boldsymbol{f}-\boldsymbol{n} \boldsymbol{\nabla} \cdot \boldsymbol{f}\right) \otimes \boldsymbol{v} & =\oint_{\partial \Sigma} \mathrm{T} \times(\boldsymbol{f} \otimes \boldsymbol{v})+\int_{\Sigma} n_{i} f_{j l} \frac{\partial v_{m}}{\partial x_{j}} \boldsymbol{e}_{i} \boldsymbol{e}_{l} \boldsymbol{e}_{m} \\
& -\int_{\Sigma} n_{i} f_{i l} \frac{\partial v_{m}}{\partial x_{j}} \boldsymbol{e}_{j} \boldsymbol{e}_{l} \boldsymbol{e}_{m} .
\end{aligned}
$$


In the above relation, replacing $\boldsymbol{f}$ by $\boldsymbol{n} \boldsymbol{f}$ leads to

$$
\begin{aligned}
\int_{\Sigma}\left(\boldsymbol{n}^{2} \cdot \nabla(\boldsymbol{n} \boldsymbol{f})-\boldsymbol{n} \nabla \cdot(\boldsymbol{n} \boldsymbol{f})\right) \otimes \boldsymbol{v} & =\oint_{\partial \Sigma} \mathrm{T} \times(\boldsymbol{n} \boldsymbol{f} \otimes \boldsymbol{v})+\int_{\Sigma} n_{i} n_{j} f_{l s} \frac{\partial v_{m}}{\partial x_{j}} \boldsymbol{e}_{i} \boldsymbol{e}_{l} \boldsymbol{e}_{s} \boldsymbol{e}_{m} \\
& -\int_{\Sigma} f_{l s} \frac{\partial v_{m}}{\partial x_{j}} \boldsymbol{e}_{j} \boldsymbol{e}_{l} \boldsymbol{e}_{s} \boldsymbol{e}_{m}
\end{aligned}
$$

After some manipulation, it can be shown that Eq. (3.34) may be written as

$$
\int_{\Sigma} \boldsymbol{L} \cdot \boldsymbol{f} \otimes \boldsymbol{v}=\oint_{\partial \Sigma} \boldsymbol{m} \cdot \boldsymbol{f} \otimes \boldsymbol{v}+\int_{\Sigma} \boldsymbol{n} \cdot \boldsymbol{f} \boldsymbol{n} \cdot \boldsymbol{\nabla} \boldsymbol{v}-\int_{\Sigma} \boldsymbol{f}^{T} \cdot \nabla \boldsymbol{v}
$$

Substituting the tensor $\stackrel{s}{\boldsymbol{\tau}}$ and vector $\boldsymbol{x}$ in place of $\boldsymbol{f}$ and $\boldsymbol{v}$, respectively, and considering the integration on the regular closed surface $\partial B$, it is inferred that

$$
\int_{\partial B} \boldsymbol{L} \cdot \stackrel{s}{\boldsymbol{\tau}} \otimes \boldsymbol{x}=-\int_{\partial B}\left(\stackrel{s}{\tau}^{T}-\boldsymbol{n} \cdot \stackrel{s}{\boldsymbol{\tau}} \boldsymbol{n}\right)
$$

Finally, Substitution of (3.31) and (3.36) into (3.29) leads to the following relation for the net surface stress on $\partial B$

$$
\int_{\partial B} \mathbf{S} \cdot \boldsymbol{P}=\int_{\partial B}\left(\boldsymbol{n} \cdot \stackrel{s}{\boldsymbol{\tau}} \boldsymbol{n}-\stackrel{s}{\boldsymbol{\tau}}^{T}\right)-\int_{B} \boldsymbol{S}^{T}+\int_{B} \boldsymbol{b}_{B} \otimes \boldsymbol{x}+\int_{\partial B} \boldsymbol{b}_{e} \otimes \boldsymbol{x}
$$

where the right hand side involves not only $\stackrel{s}{\boldsymbol{\tau}}$ on $\partial B$, but also $\stackrel{1}{\boldsymbol{\tau}}, \stackrel{2}{\boldsymbol{\tau}}$, and $\stackrel{3}{\boldsymbol{\tau}}$ within B.

\subsection{Energy considerations}

According to Mindlin (1965), the bulk elastic strain energy of an isotropic, centrosymmetric, and elastic material occupying the region, $B$ corresponding to the displacement field, $\boldsymbol{u}$ is

$$
U_{B}=\frac{1}{2} \int_{B}(\stackrel{1}{\boldsymbol{\tau}}: \nabla \boldsymbol{u}+\stackrel{2}{\boldsymbol{\tau}}: \boldsymbol{\nabla} \boldsymbol{\nabla} \boldsymbol{u}+\stackrel{3}{\boldsymbol{\tau}}:: \nabla \nabla \nabla \boldsymbol{\nabla} \boldsymbol{u})+\frac{1}{2} b_{0} \int_{B} \nabla^{2} \boldsymbol{\nabla} . \boldsymbol{u} .
$$


In addition, due to the elastic model of Gurtin and Murdoch (1975), the elastic strain energy of surface $\partial B$ pertinent to the displacement field, $\boldsymbol{u}$ and surface strain, $\mathbf{E}$ is

$$
U_{\partial B}=\frac{1}{2} \int_{\partial B}\left((\mathbf{C}: \mathbf{E}): \mathbf{E}+\left(\left(\boldsymbol{u} \overleftarrow{\nabla}_{\jmath}\right) \cdot \mathbf{M}\right): \boldsymbol{u} \overleftarrow{\nabla}_{\jmath}\right)
$$

Theorem 3.1. Let $\boldsymbol{u}$ be a solution for a three-dimensional body $B$ with boundary $\partial B$ and unit outward normal vector $\boldsymbol{n}$. Then, the total elastic energy is given by

$$
\begin{aligned}
U_{B}+U_{\partial B} & =-\frac{1}{2} \int_{\partial B} \mathbf{M}: \mathbf{E}+\frac{b_{0}}{2} \int_{B} \nabla^{2} \nabla \cdot \boldsymbol{u}+\frac{1}{2} \int_{\partial B}\left(\boldsymbol{b}_{e} \cdot \boldsymbol{u}+\stackrel{2}{\boldsymbol{t}} \cdot D \boldsymbol{u}+\stackrel{3}{\boldsymbol{t}} \cdot D^{2} \boldsymbol{u}\right) \\
& +\frac{1}{2} \int_{B} \boldsymbol{b}_{B} \cdot \boldsymbol{u} .
\end{aligned}
$$

Proof. By using divergence theorem, it is readily shown that

$$
\int_{\partial B} \boldsymbol{n} \cdot \boldsymbol{S} \cdot \boldsymbol{u}=\int_{B} \boldsymbol{\nabla} \boldsymbol{u}: \boldsymbol{S}-\int_{B} \boldsymbol{b}_{B} \cdot \boldsymbol{u} .
$$

Next, by using Eq. (3.9a), after some manipulation, we obtain

$$
\begin{aligned}
\int_{\partial B} \boldsymbol{n} . \boldsymbol{S} \cdot \boldsymbol{u} & =\int_{B}(\boldsymbol{\nabla u}: \stackrel{1}{\boldsymbol{\tau}}+\nabla \nabla \boldsymbol{u}: \stackrel{2}{\boldsymbol{\tau}}+\nabla \nabla \nabla \boldsymbol{u}:: \stackrel{3}{\boldsymbol{\tau}}) \\
& +\int_{\partial B} \boldsymbol{\nabla} \boldsymbol{u}:(\boldsymbol{n} \cdot \boldsymbol{\nabla} \cdot \stackrel{3}{\boldsymbol{\tau}}-\boldsymbol{n} \cdot \stackrel{2}{\boldsymbol{\tau}})-\int_{\partial B} \nabla \nabla \boldsymbol{u}:(\boldsymbol{n} \cdot \stackrel{3}{\boldsymbol{\tau}})-\int_{B} \boldsymbol{b}_{B} \cdot \boldsymbol{u}
\end{aligned}
$$

and employing (3.38)

$$
\begin{aligned}
\int_{\partial B} \boldsymbol{n} \cdot \boldsymbol{S} \cdot \boldsymbol{u} & =2 U_{B}-b_{0} \int_{B} \nabla^{2} \nabla \cdot \boldsymbol{u}+\int_{\partial B} \boldsymbol{\nabla} \boldsymbol{u}:(\boldsymbol{n} \cdot \boldsymbol{\nabla} \cdot \stackrel{3}{\boldsymbol{\tau}}-\boldsymbol{n} \cdot \stackrel{2}{\boldsymbol{\tau}})^{2} \\
& -\int_{\partial B} \nabla \nabla \boldsymbol{u}:(\boldsymbol{n} \cdot \stackrel{3}{\boldsymbol{\tau}})-\int_{B} \boldsymbol{b}_{B} \cdot \boldsymbol{u} .
\end{aligned}
$$

The gradient of the displacement vector is splitted into the surface gradient and normal gradient and then the surface divergence theorem (2.8) for regular closed 
surface $\partial B$ is utilized to get

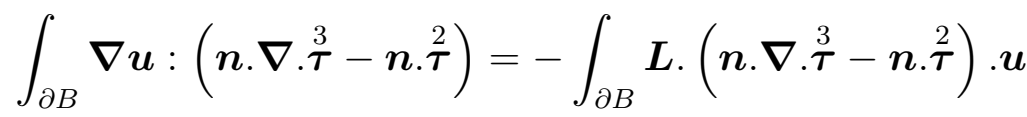

$$
\begin{aligned}
& +\int_{\partial B} \boldsymbol{n} \cdot\left(\boldsymbol{n} \cdot \boldsymbol{\nabla} \cdot \mathrm{\tau}^{3}-\boldsymbol{n} \cdot \mathbf{\tau}^{2}\right) \cdot D \boldsymbol{u}
\end{aligned}
$$

In a similar manner

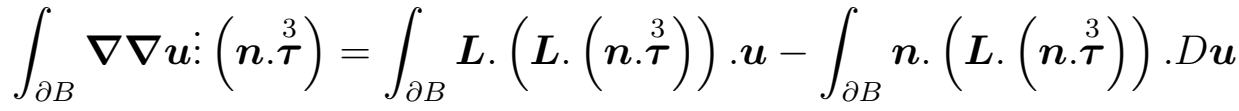

$$
\begin{aligned}
& +\int_{\partial B}\left(\boldsymbol{n} \boldsymbol{n}: \stackrel{3}{\boldsymbol{\tau}}^{2}\right): D \boldsymbol{\nabla} \boldsymbol{u}
\end{aligned}
$$

Noting that $D \boldsymbol{\nabla} \boldsymbol{u}=\mathbf{I} \cdot \nabla_{\jmath} D \boldsymbol{u}+\boldsymbol{n} D^{2} \boldsymbol{u}-(\boldsymbol{\nabla} \boldsymbol{n}) . \boldsymbol{\nabla} \boldsymbol{u}$ and $\boldsymbol{\nabla} \boldsymbol{n}=\mathbf{I} \cdot \boldsymbol{\nabla}_{\jmath} \boldsymbol{n}$, manipulation of the last integral of Eq. (3.45) for closed regular surface $\partial B$ results in

$$
\begin{aligned}
& \int_{\partial B}(\boldsymbol{n} \boldsymbol{n}: \stackrel{3}{\boldsymbol{\tau}}): D \boldsymbol{\nabla} \boldsymbol{u}=\int_{\partial B}\left(-\boldsymbol{L} \cdot\left(\boldsymbol{n n}: \stackrel{3}{\boldsymbol{\tau}}^{2}\right) \cdot D \boldsymbol{u}+\boldsymbol{L} \cdot\left(\left(\mathbf{I} \cdot \boldsymbol{\nabla}_{\jmath} \boldsymbol{n}\right) \cdot\left(\boldsymbol{n} \boldsymbol{n}: \stackrel{3}{\boldsymbol{\tau}}^{2}\right)\right) \cdot \boldsymbol{u}\right. \\
& \left.+\boldsymbol{n n n}: \frac{3}{\tau} \cdot D^{2} \boldsymbol{u}\right) \text {. }
\end{aligned}
$$

Therefore, relation (3.43) can be written as

$$
\int_{\partial B} \boldsymbol{n} . \boldsymbol{S} \cdot \boldsymbol{u}=2 U_{B}-b_{0} \int_{B} \nabla^{2} \boldsymbol{\nabla} \cdot \boldsymbol{u}-\int_{\partial B}\left(-\boldsymbol{L} \cdot \stackrel{s}{\boldsymbol{\tau}} \cdot \boldsymbol{u}+\stackrel{2}{\boldsymbol{t}} \cdot D \boldsymbol{u}+\stackrel{3}{\boldsymbol{t}} \cdot D^{2} \boldsymbol{u}\right)-\int_{B} \boldsymbol{b}_{B} \cdot \boldsymbol{u} .
$$

Moreover, employing Eqs. (3.8a), (3.17), and (3.16), it is deduced that for the closed regular surface $\partial B$

$$
\int_{\partial B} \boldsymbol{n} \cdot \boldsymbol{S} \cdot \boldsymbol{u}=-\int_{\partial B} \mathbf{S}: \boldsymbol{u} \overleftarrow{\boldsymbol{\nabla}}_{\jmath}+\int_{\partial B} \boldsymbol{L} \cdot \stackrel{s}{\boldsymbol{\tau}} \cdot \boldsymbol{u}+\int_{\partial B} \boldsymbol{b}_{e} \cdot \boldsymbol{u} \cdot
$$

After insertion of the expression for $\mathbf{S}$ from Eq. (3.20) into Eq. (3.48), the following relation is obtained

$$
\int_{\partial B} \boldsymbol{n} \cdot \boldsymbol{S} \cdot \boldsymbol{u}=-\int_{\partial B}\left(\mathbf{M}: \mathbf{E}+(\mathbf{C}: \mathbf{E}): \mathbf{E}+\left(\left(\boldsymbol{u} \overleftarrow{\nabla}_{\jmath}\right) \cdot \mathbf{M}\right): \boldsymbol{u} \overleftarrow{\nabla}_{\jmath}\right)
$$




$$
+\int_{\partial B} \boldsymbol{L} \cdot \stackrel{s}{\boldsymbol{\tau}} \cdot \boldsymbol{u}+\int_{\partial B} \boldsymbol{b}_{e} \cdot \boldsymbol{u}
$$

or equivalently

$$
\int_{\partial B} \boldsymbol{n} \cdot \boldsymbol{S} \cdot \boldsymbol{u}=-2 U_{\partial B}-\int_{\partial B} \mathbf{M}: \mathbf{E}+\int_{\partial B} \boldsymbol{L} \cdot \stackrel{s}{\boldsymbol{\tau}} \cdot \boldsymbol{u}+\int_{\partial B} \boldsymbol{b}_{e} \cdot \boldsymbol{u} .
$$

Comparison of Eqs. (3.47) and (3.50) completes the proof.

\section{Film of infinite extension}

In this section, utilizing the present theory, the exact analytical expressions for the Piola-Kirchhoff surface stress tensor, tangential residual surface stress tensor, surface elastic constants, and effective elastic constants associated with an infinitely extended planar film of thickness, $t$ are derived. Place the origin of the Cartesian coordinates midway through the thickness of the film and choose the coordinate axes in such a way that $-\infty<x_{2}, x_{3}<\infty$ and $\left|x_{1}\right| \leq t / 2$. For the time being, suppose that the film is subjected to a combined uniform biaxial tension and uniform shear stress, then the pertaining displacement field is of the form

$$
\boldsymbol{u}=\left(A_{1}\left(x_{1}\right), A_{2} x_{2}+A_{4} x_{3}, A_{3} x_{3}+A_{4} x_{2}\right),
$$

where $A_{2}, A_{3}$, and $A_{4}$ are constant. The corresponding expressions for $\stackrel{1}{\boldsymbol{\epsilon}}, \stackrel{2}{\boldsymbol{\epsilon}}$, and $\stackrel{3}{\boldsymbol{\epsilon}}$ are obtained from Eqs. (3.1). Subsequently, with due attention to Eqs. (3.13) and (3.5), the expressions for $\stackrel{1}{\boldsymbol{\tau}}, \underset{\boldsymbol{\tau}}{\boldsymbol{\tau}}$, and $\stackrel{3}{\boldsymbol{\tau}}$ are derived. Finally, by consideration of Eqs. (3.9a) and (3.7), the displacement formulation of the equilibrium is found. The 
generalized surface tractions, $\stackrel{1}{\boldsymbol{t}}, \stackrel{2}{\boldsymbol{t}}$, and $\stackrel{3}{\boldsymbol{t}}$ given in Eqs. (3.8) may also be represented in terms of the displacements. As noted earlier, for simplicity, only the second order tangential surface strain tensor, $\mathbf{E}=\stackrel{1}{\mathbf{E}}$ is maintained. The Piola-Kirchhoff surface stress tensor, S is calculated by virtue of Eqs. (3.2) and (3.20). For the considered planar film, the unit outward normal vectors to the top and bottom surfaces of the film are $\boldsymbol{n}=( \pm 1,0,0)$ and hence, from the definition of the perpendicular projection, $\boldsymbol{P}$ and Eq. (2.1), we have

$$
\boldsymbol{P}=\left(\begin{array}{lll}
0 & 1 & 0 \\
0 & 0 & 1
\end{array}\right), \quad \mathbf{I}=\left(\begin{array}{ll}
0 & 0 \\
1 & 0 \\
0 & 1
\end{array}\right) .
$$

Subsequently, the surface tangential strain tensor and Piola-Kirchhoff surface stress tensor throughout either the top or bottom surfaces of the film are

$$
\begin{aligned}
\mathbf{E}=\left(\begin{array}{ll}
A_{2} & A_{4} \\
A_{4} & A_{3}
\end{array}\right), \\
\mathbf{S}=\left(\begin{array}{cc}
\mathrm{M}_{22}+A_{2} \mathrm{M}_{22}+A_{4} \mathrm{M}_{32} & \mathrm{M}_{23}+A_{2} \mathrm{M}_{23}+A_{4} \mathrm{M}_{33} \\
0 & 0 \\
\mathrm{M}_{32}+A_{4} \mathrm{M}_{22}+A_{3} \mathrm{M}_{32} & \mathrm{M}_{33}+A_{4} \mathrm{M}_{23}+A_{3} \mathrm{M}_{33}
\end{array}\right) \\
+\left(\begin{array}{cc}
0 & 0 \\
\mathrm{C}_{2222} A_{2}+\mathrm{C}_{2233} A_{3}+2 \mathrm{C}_{2223} A_{4} & \mathrm{C}_{2322} A_{2}+2 \mathrm{C}_{2323} A_{4}+\mathrm{C}_{2333} A_{3} \\
\mathrm{C}_{2322} A_{2}+2 \mathrm{C}_{2323} A_{4}+\mathrm{C}_{2333} A_{3} & \mathrm{C}_{2233} A_{2}+2 \mathrm{C}_{2333} A_{4}+\mathrm{C}_{3333} A_{3}
\end{array}\right),
\end{aligned}
$$


respectively, where $M_{22}, M_{23}=M_{32}$, and $M_{33}$ are the components of the symmetric residual tangential surface stress tensor merely due to the free surface effects of the film. From Eq. (4.3b), it is observed that for the infinitely extended homogeneous film with constant surface elastic moduli tensor and residual tangential surface stress tensor throughout the film surfaces, the surface Piola-Kirchhoff stress tensor is constant, and hence $\operatorname{div}_{s} \mathbf{S}=\mathbf{0}$ which in turn, according to Eq. (3.16), results in $\boldsymbol{b}_{\jmath}=\mathbf{0}$. In the absence of surface body forces, $\boldsymbol{b}_{s}$ and $\boldsymbol{b}_{e}$, Eq. (3.17) leads to $\boldsymbol{t}=\mathbf{0}$ on the film surfaces. Moreover, for the supposed displacement field for the infinitely extended film $\operatorname{div}_{\jmath}\left(\mathbf{M} \cdot\left(\vec{\nabla}_{\jmath} \boldsymbol{u}\right) \cdot \boldsymbol{n}\right)=0$, and hence, zero surface curvature tensor, $\boldsymbol{\kappa}=\mathbf{0}$ for the film surfaces satisfies Eq. (3.25). Assuming that the film surfaces are traction-free, the generalized surface tractions $\stackrel{2}{\boldsymbol{t}}$ and $\stackrel{3}{\boldsymbol{t}}$ are equal to zero. By solving the equilibrium equations (3.7) with $\boldsymbol{b}_{B}=\mathbf{0}$ and $\stackrel{1}{\boldsymbol{t}}=\stackrel{2}{\boldsymbol{t}}=\stackrel{3}{\boldsymbol{t}}=\mathbf{0}$, the following result is obtained

$$
\begin{aligned}
A_{1}\left(x_{1}\right) & =-\left(A_{2}+A_{3}\right)\left(\frac{\lambda x_{1}}{\lambda+2 \mu}+\left(\lambda \ell_{10}^{2}-c_{1}\right)\left(C_{1}^{*} \sinh \left(x_{1} / \ell_{11}\right)+C_{2}^{*} \sinh \left(x_{1} / \ell_{12}\right)\right)\right) \\
& +u^{*}\left(x_{1}\right)
\end{aligned}
$$

where

$$
u^{*}\left(x_{1}\right)=b_{0}\left(C_{1}^{*} \sinh \left(x_{1} / \ell_{11}\right)+C_{2}^{*} \sinh \left(x_{1} / \ell_{12}\right)\right)
$$


is due to the surface effect solely. In the above relations

$$
\begin{gathered}
C_{1}^{*}=\frac{-\ell_{11}^{2}\left(\ell_{10}^{2}+\ell_{12}^{2}\right) / \sinh \frac{t}{2 \ell_{11}}}{(\lambda+2 \mu)\left(\ell_{11}\left(\ell_{10}^{2}+\ell_{12}^{2}\right)^{2} \operatorname{coth} \frac{t}{2 \ell_{11}}-\ell_{12}\left(\ell_{10}^{2}+\ell_{11}^{2}\right)^{2} \operatorname{coth} \frac{t}{2 \ell_{12}}\right)}, \\
C_{2}^{*}=\frac{\ell_{12}^{2}\left(\ell_{10}^{2}+\ell_{11}^{2}\right) / \sinh \frac{t}{2 \ell_{12}}}{(\lambda+2 \mu)\left(\ell_{11}\left(\ell_{10}^{2}+\ell_{12}^{2}\right)^{2} \operatorname{coth} \frac{t}{2 \ell_{11}}-\ell_{12}\left(\ell_{10}^{2}+\ell_{11}^{2}\right)^{2} \operatorname{coth} \frac{t}{2 \ell_{12}}\right)},
\end{gathered}
$$

and the surface characteristic length, $\ell_{10}$ is defined as

$$
(\lambda+2 \mu) \ell_{10}^{2}=\bar{c}
$$

The total elastic energy of the film per unit area of the film surface is calculated from Eq. (3.40) as below

$$
\begin{aligned}
U_{B}+U_{\partial B} & =2 t \mu\left(A_{4}^{2}+\frac{(\lambda+\mu)\left(A_{2}^{2}+A_{3}^{2}\right)+\lambda A_{2} A_{3}}{\lambda+2 \mu}\right)-\frac{\bar{\sigma} b_{0}}{2}\left(A_{2}+A_{3}\right) \\
& +b_{0}\left(b_{0}-\left(\lambda \ell_{10}^{2}-c_{1}\right)\left(A_{2}+A_{3}\right)\right)\left(\frac{C_{1}^{*}}{\ell_{11}^{2}} \sinh \frac{t}{2 \ell_{11}}+\frac{C_{2}^{*}}{\ell_{12}^{2}} \sinh \frac{t}{2 \ell_{12}}\right) .
\end{aligned}
$$

It is interesting to note that, in Eq. (4.4), except for the first term, all the remaining terms stem solely due to the second strain gradient theory. Thus, the solution of this problem in the context of surface elasticity presented by Gurtin and Murdoch (1975) is readily obtained from the above-mentioned solution if one ignores the terms containing Mindlin's additional elastic constants and characteristic lengths

$$
\boldsymbol{u}=\left(\frac{-\lambda\left(A_{2}+A_{3}\right) x_{1}}{\lambda+2 \mu}, A_{2} x_{2}+A_{4} x_{3}, A_{3} x_{3}+A_{4} x_{2}\right)
$$


This displacement field results in the following uniform stresses within the film

$$
\begin{aligned}
& \tau_{22}=\frac{4 \mu(\mu+\lambda) A_{2}+2 \lambda \mu A_{3}}{\lambda+2 \mu}, \\
& \tau_{33}=\frac{4 \mu(\mu+\lambda) A_{3}+2 \lambda \mu A_{2}}{\lambda+2 \mu}, \\
& \tau_{23}=\tau_{32}=2 \mu A_{4},
\end{aligned}
$$

which clearly correspond to $\stackrel{1}{\tau}$.

For the special case where the in-plane loadings are absent, $A_{2}=A_{3}=A_{4}=0$. Consequently, Eq. (4.1) reduces to $\boldsymbol{u}=\left(u^{*}\left(x_{1}\right), 0,0\right)$, and the surface tangential strain tensor, $\mathbf{E} \equiv 0$. For thin films, the scenario where the surface effect is the only root cause of the displacement field $\boldsymbol{u}=\left(u^{*}\left(x_{1}\right), 0,0\right)$ has been discussed by Ojaghnezhad and Shodja (2013) in the mathematical framework of Mindlin's second strain gradient elasticity. This nontrivial solution is due to the consideration of the modulus of cohesion, $b_{0}$ in the second strain gradient theory. In this case, the only non-zero component of the strain tensor is

$$
\epsilon_{11}^{*}=b_{0}\left(\frac{C_{1}^{*}}{\ell_{11}} \cosh \frac{x_{1}}{\ell_{11}}+\frac{C_{2}^{*}}{\ell_{12}} \cosh \frac{x_{1}}{\ell_{12}}\right) .
$$

Clearly, if $b_{0}=0$, then only trivial solution prevails since according to Eqs. (4.5) and (4.11), both $u^{*}\left(x_{1}\right)$ and $\epsilon_{11}^{*}$ vanish. It should be emphasized that for the considered film, both Gurtin and Murdoch (1975)'s surface elasticity and Mindlin's first strain gradient elasticity lead to the trivial solution when the in-plane loadings are absent. 
4.1. Residual surface stress tensor and surface elastic constants in the mathematical framework of second strain gradient theory

As it was alluded to, the mathematical framework of Gurtin and Murdoch (1975) surface elasticity gives rise to residual surface stress tensor and surface elastic constants. The relation between these surface parameters and Mindlin's additional constants in second strain gradient elasticity pertinent to the film under consideration is of particular interest. By using the displacement field (4.1), the generalized strain and stress tensors are calculated via Eqs. (3.1) and (3.5), respectively. Sub-

sequently, $\stackrel{s}{\boldsymbol{\tau}}$ is obtained from Eq. (3.9b). It is observed that for the considered displacement field, the nonzero components of $\stackrel{s}{\boldsymbol{\tau}}$ on $x_{1}= \pm t / 2$ are $\stackrel{s}{\tau} 11$ and $\stackrel{s}{\tau}{ }_{22}=\stackrel{s}{\tau}_{33}$ which are constant within the top and bottom surfaces. To simulate the infinitely extended film under uniform in-plane loadings, it is assumed that on the film surfaces with unit normal vectors $(0, \pm 1,0)$ and $(0,0, \pm 1)$ at infinity, the surface body force $\boldsymbol{b}_{e}=\boldsymbol{n} . \boldsymbol{\tau}$ is applied, where $\boldsymbol{\tau}$ is obtained from Eqs. (4.10). Noting that $\mathbf{S}$ is constant within the film surfaces, $x_{1}= \pm t / 2$, by employing the relation (3.37) one may obtain the nonzero components of the surface stress tensor as below

$$
\begin{aligned}
& \mathrm{S}_{22}=\mathrm{S}_{33}=\bar{\sigma}\left[b_{0}-\left(A_{2}+A_{3}\right)\left(\lambda \ell_{10}^{2}-c_{1}\right)\right], \\
& \mathrm{S}_{23}=\mathrm{S}_{32}=0
\end{aligned}
$$

where

$$
\bar{\sigma}=\frac{\left(c_{1}-\lambda \ell_{10}^{2}\right)\left(\ell_{12}^{2}-\ell_{11}^{2}\right) /(\lambda+2 \mu)}{\ell_{11}\left(\ell_{10}^{2}+\ell_{12}^{2}\right)^{2} \operatorname{coth} \frac{t}{2 \ell_{11}}-\ell_{12}\left(\ell_{10}^{2}+\ell_{11}^{2}\right)^{2} \operatorname{coth} \frac{t}{2 \ell_{12}}} .
$$


With due attention to Eq. (4.3b) and Eqs. (4.12) and assuming an arbitrary choice of constants $A_{2}, A_{3}$, and $A_{4}$, the components of the residual surface stress tensor, $\mathbf{M}$ and surface elastic constants, $\mathbf{C}$ are obtained as

$$
\begin{aligned}
& \mathrm{M}_{22}=\mathrm{M}_{33}=b_{0} \bar{\sigma}, \\
& \mathrm{M}_{23}=\mathrm{M}_{32}=0,
\end{aligned}
$$

and

$$
\begin{aligned}
& \mathrm{C}_{2222}=\mathrm{C}_{3333}=-\bar{\sigma}\left(b_{0}+\lambda \ell_{10}^{2}-c_{1}\right), \\
& \mathrm{C}_{2233}=-\bar{\sigma}\left(\lambda \ell_{10}^{2}-c_{1}\right), \\
& \mathrm{C}_{2323}=-\frac{b_{0} \bar{\sigma}}{2}, \\
& \mathrm{C}_{2322}=\mathrm{C}_{2333}=0,
\end{aligned}
$$

respectively. As noted by Mindlin (1965), the modulus of cohesion, $b_{0}$ which is the coefficient of the term linear in $\stackrel{3}{\boldsymbol{\epsilon}}$ in the expression (3.13) for the strain energy density gives rise to self-equilibrating components of cohesive force, and has dimension of force. In the context of the present formulation, as it is seen from Eq. (4.14a), modulus of cohesion is related to the normal components of the residual surface stress tensor (surface tension). For a detailed discussion on the physical interpretation and the origin of surface tension as well as the source of confusion between the notions of surface tension and surface energy, the reader may refer to the work of Shuttleworth (1950). An important note emphasized by Shuttleworth (1950) is that, although surface tension and surface energy have the same dimension, they are physically 
different entities. These parameters become equivalent in the special case of onecomponent liquids.

From Eqs. (4.15), it is observed that the requirement for isotropic surfaces, $\mathrm{C}_{2323}=\left(\mathrm{C}_{2222}-\mathrm{C}_{2233}\right) / 2$ is satisfied. In view of Eqs. (3.21), (4.14), and (4.15), it is deduced that for isotropic surfaces

$$
\begin{aligned}
& \sigma_{0}=b_{0} \bar{\sigma}, \\
& \lambda_{0}=\mathrm{C}_{2233}, \\
& \mu_{0}=\mathrm{C}_{2323},
\end{aligned}
$$

and hence, $\mathrm{C}_{2222}=\mathrm{C}_{3333}=\lambda_{0}+2 \mu_{0}$. It should also be noted that the residual surface stress tensor, $\mathbf{M}$ takes the form of isotropic surface tension. Moreover, since $\bar{\sigma}$ depends on the film thickness, then both the residual surface stress and surface elastic constants depend on the film thickness. However, if the thickness is large in comparison to the characteristic lengths $\ell_{11}$ and $\ell_{12}$, then the expression for $\bar{\sigma}$ reduces to the following form

$$
\bar{\sigma}=\frac{\left(c_{1}-\lambda \ell_{10}^{2}\right)\left(\ell_{12}^{2}-\ell_{11}^{2}\right) /(\lambda+2 \mu)}{\ell_{11}\left(\ell_{10}^{2}+\ell_{12}^{2}\right)^{2}-\ell_{12}\left(\ell_{10}^{2}+\ell_{11}^{2}\right)^{2}},
$$

which no longer varies with the film thickness.

\subsection{The overall elastic constants}

In this section, an estimation for the overall elastic constants of the film including the surface effect is pursued. As it was alluded to, even in the absence of external 
loadings, a nontrivial solution within the film exists merely due to the surface effect. This effect gives rise to the change in the film thickness, $\Delta t$ according to the following relation

$$
\Delta t=\frac{2 b_{0} \ell_{10}^{2}\left(\ell_{12}^{2}-\ell_{11}^{2}\right)}{(\lambda+2 \mu)\left(\ell_{11}\left(\ell_{10}^{2}+\ell_{12}^{2}\right)^{2} \operatorname{coth} \frac{t}{2 \ell_{11}}-\left(\ell_{10}^{2}+\ell_{11}^{2}\right)^{2} \ell_{12} \operatorname{coth} \frac{t}{2 \ell_{12}}\right)}
$$

Therefore, the average longitudinal strain in the direction of $x_{1}$ over the film thickness stemming from the surface effect measures as $\bar{\epsilon}_{11}^{*}=\Delta t / t$. Moreover, the resulting average residual stresses along the film thickness are

$$
\bar{\tau}_{22}^{*}=\bar{\tau}_{33}^{*}=\frac{\lambda \ell_{10}^{2}-c_{1}}{\ell_{10}^{2}} \frac{\Delta t}{t} .
$$

To estimate the effective elastic constants $C_{1111}^{\text {eff }}$ and $C_{2211}^{\text {eff }}$, we first allow the film to relax and reach its equilibrium deformed shape under the surface effect. Then, an additional external uniform uniaxial strain loading, $A_{1}$ is applied. Therefore, the total average uniaxial strain is

$$
\bar{\epsilon}_{11}=A_{1}\left(1+\bar{\epsilon}_{11}^{*}\right)
$$

The resulting average stress components in the directions $x_{1}$ and $x_{2}$ obtained from classical effective constitutive law are made equivalent to the average of those obtained from second strain gradient theory as below

$$
\begin{aligned}
& C_{1111}^{\mathrm{eff}} \bar{\epsilon}_{11}=A_{1}(\lambda+2 \mu), \\
& C_{2211}^{\mathrm{eff}} \bar{\epsilon}_{11}=A_{1}\left(\lambda+\frac{\lambda \ell_{10}^{2}-c_{1}}{\ell_{10}^{2}} \frac{\Delta t}{t}\right),
\end{aligned}
$$


which lead to

$$
\begin{aligned}
& C_{1111}^{\mathrm{eff}}=\frac{\lambda+2 \mu}{1+\Delta t / t} \\
& C_{2211}^{\mathrm{eff}}=\lambda-\frac{c_{1}}{\ell_{10}^{2}} \frac{\Delta t / t}{1+\Delta t / t} .
\end{aligned}
$$

It is noteworthy to mention that for large enough thicknesses of the film $\operatorname{coth} \frac{t}{2 \ell_{11}} \rightarrow$ 1 and $\operatorname{coth} \frac{t}{2 \ell_{12}} \rightarrow 1$ and hence according to Eqs. (4.18) and (4.22), $C_{1111}^{\mathrm{eff}} \rightarrow \lambda+2 \mu$ and $C_{2211}^{\text {eff }} \rightarrow \lambda$. Note that due to the symmetry $C_{3311}^{\text {eff }}=C_{2211}^{\text {eff }}$. However, as it will be shown shortly, $C_{1122}^{\mathrm{eff}} \neq C_{2211}^{\mathrm{eff}}$ for small thicknesses of the film. To determine $C_{2222}^{\mathrm{eff}}$ and $C_{1122}^{\text {eff }}$, the displacement field (4.1) with $A_{3}=A_{4}=0$ is applied to the film. For this type of loading, the averages of the nonzero normal strains are as below

$$
\begin{aligned}
& \bar{\epsilon}_{11}=-\frac{\lambda A_{2}}{\lambda+2 \mu}-A_{2} \frac{\lambda \ell_{10}^{2}-c_{1}}{b_{0}} \frac{\Delta t}{t}+\bar{\epsilon}_{11}^{*}, \\
& \bar{\epsilon}_{22}=A_{2} .
\end{aligned}
$$

The equivalency between the components of the stresses obtained from the effective constitutive relation and second strain gradient theory yield

$$
\begin{aligned}
& C_{1122}^{\mathrm{eff}} \bar{\epsilon}_{22}+C_{1111}^{\mathrm{eff}}\left(\bar{\epsilon}_{11}-\bar{\epsilon}_{11}^{*}\right)=0 \\
& C_{2222}^{\mathrm{eff}} \bar{\epsilon}_{22}+C_{2211}^{\mathrm{eff}}\left(\bar{\epsilon}_{11}-\bar{\epsilon}_{11}^{*}\right)=\frac{4 \mu(\lambda+\mu) A_{2}}{\lambda+2 \mu}-\frac{\left(\lambda \ell_{10}^{2}-c_{1}\right)^{2}}{b_{0} \ell_{10}^{2}} \frac{\Delta t}{t} A_{2},
\end{aligned}
$$

resulting in

$$
\begin{aligned}
& C_{1122}^{\mathrm{eff}}=\frac{\lambda}{1+\Delta t_{\mathrm{f}} / t}+(\lambda+2 \mu) \frac{\lambda \ell_{10}^{2}-c_{1}}{b_{0}} \frac{\Delta t / t}{1+\Delta t / t} \\
& C_{2222}^{\mathrm{eff}}=\lambda+2 \mu+\frac{c_{1}}{\ell_{10}^{2}}\left(\frac{\lambda \ell_{10}^{2}-c_{1}}{b_{0}}-\frac{\lambda}{\lambda+2 \mu}\right) \frac{\Delta t / t}{1+\Delta t / t} .
\end{aligned}
$$


From Eqs. (4.22b) and (4.25a) it is seen that $C_{1122}^{\text {eff }} \neq C_{2211}^{\text {eff }}$ - a manifestation of surface effect which clearly becomes important for small thicknesses. It is readily observed that for large enough thickness of the film, $C_{1122}^{\mathrm{eff}} \rightarrow \lambda$ and $C_{2222}^{\mathrm{eff}} \rightarrow \lambda+2 \mu$. Moreover, $C_{1133}^{\text {eff }}=C_{1122}^{\text {eff }}$ and $C_{3333}^{\text {eff }}=C_{2222}^{\text {eff }}$ in view of the symmetry considerations. To derive the expression for $C_{2233}^{\text {eff }}$, the displacement field (4.1) with $A_{4}=0$ is employed. Following a similar procedure as explained above, it is obtained that

$$
C_{2233}^{\mathrm{eff}}=C_{3322}^{\mathrm{eff}}=\lambda+\frac{c_{1}}{\ell_{10}^{2}}\left(\frac{\lambda \ell_{10}^{2}-c_{1}}{b_{0}}-\frac{\lambda}{\lambda+2 \mu}\right) \frac{\Delta t / t}{1+\Delta t / t} .
$$

\subsection{Calculation of surface characteristic length and modulus of cohesion}

As noted in Section 3.4 on energy considerations, the general expression for the total elastic strain energy via surface elasticity reformulated in the context of second strain gradient theory is given by (3.40); it should be noted that in the absence of any external loadings it reduces to

$$
U_{B}+U_{\partial B}=-\frac{1}{2} \int_{\partial B} \mathbf{M}: \mathbf{E}+\frac{b_{0}}{2} \int_{B} \nabla^{2} \nabla \cdot \boldsymbol{u}
$$

which inherits the physical interpretations given in the works of both Gurtin and Murdoch (1975) and Mindlin (1965). In the above relation, the first and second terms stem from the presence of surface strain tensor, $\mathbf{E}$ and the modulus of cohesion, $b_{0}$, respectively. In general, the first term which is due to surface elasticity theory survives only if the projection of $\boldsymbol{u} \overleftarrow{\nabla}_{s}$ on the surface and subsequently, the surface strain tensor, E given by Eq. (3.2) is nonzero. On the other hand, the second term has entered the formulation from consideration of the second strain gradient 
theory and, in general, is nonzero due to the modulus of cohesion. Mindlin (1965) considered a free-standing half-space and obtained an expression for the surface energy in terms of modulus of cohesion. Recently, Ojaghnezhad and Shodja (2013) expressed the surface energy in terms of modulus of cohesion by analyzing a freestanding thin films; they calculated the involved second strain gradient characteristic lengths with the aids of first principles calculations. In the current work, the surface energy for the free-standing film can be obtained by setting $A_{2}=A_{3}=A_{4}=0$ in Eq. (4.8); under this condition, the surface strain tensor is identically equal to zero, and so the surface energy, $\gamma$ in the context of second strain gradient is related to modulus of cohesion as

$$
\gamma=b_{0}^{2}\left(\frac{C_{1}^{*}}{\ell_{11}^{2}} \sinh \frac{t}{2 \ell_{11}}+\frac{C_{2}^{*}}{\ell_{12}^{2}} \sinh \frac{t}{2 \ell_{12}}\right)
$$

Moreover, for the free-standing film, the surface stress tensor given by Eqs. (4.12) represents the surface residual stresses (surface tension) as given by Eqs. (4.14). The available experimental works on the surface energy and residual surface stresses have not examined the effect of the film thickness on these parameters. Utilization of the available experimental values of these parameters and neglecting the effect of film thickness, Eqs. (4.14a) and (4.28) lead to two equations for $c_{1}, b_{0}, \ell_{10}, \ell_{11}$, $\ell_{12}, \lambda$, and $\mu$. But, the bulk characteristic lengths, $\ell_{11}$ and $\ell_{12}$ as well as Lamé constants, $\lambda$ and $\mu$ are calculated based on some formulations derived from the atomistic consideration; the details of the formulations and discussion are given in the next section. Subsequently, their numerical values are obtained via ab initio 
calculations in Section 6. Thus, by employing the approximation $c_{1}=(\lambda+2 \mu) \ell_{10}^{2} / 3$, Eqs. (4.14a) and (4.28) boil down to two equations for modulus of cohesion, $b_{0}$ and surface characteristic length, $\ell_{10}$. It is noteworthy to mention that the surface parameter, $\ell_{10}$ and modulus of cohesion, $b_{0}$ are influenced by the internal length scales of the crystalline material. Moreover, the distinction between the surface tension and surface energy, as emphasized by Shuttleworth (1950), is clearly observed from Eqs. (4.14a) and (4.28).

\section{First principles calculations of Lamé constants and bulk characteristic lengths}

In this section, for centrosymmetric crystals, an atomistic consideration is presented to link the pertinent Lamé constants and bulk characteristic lengths, $\ell_{11}$, $\ell_{12}, \ell_{21}$, and $\ell_{22}$ with the atomic force constants as defined in the context of lattice dynamics. Employing these relations and the atomic force constants obtained from ab initio calculations, one would be able to evaluate Lamé constants and bulk characteristic lengths of the crystal.

\subsection{Atomistic consideration}

Let $\boldsymbol{x}$ denote the position of an arbitrary primitive unit cell within the bulk of a centrosymmetric crystal with perfect lattice of infinite extension in space. Furthermore, suppose that the distance between the mentioned primitive unit cell and the $\alpha^{\text {th }}$-unit cell is indicated as $\boldsymbol{R}_{\alpha}$. If $K_{i j}^{\alpha}$ is the atomic force constant between the 
unit cells which are located at $\boldsymbol{x}$ and $\boldsymbol{x}+\boldsymbol{R}_{\alpha}$ and, moreover, $v$ is the volume of the primitive unit cell, then for any perturbation of the atomic arrangement from the equilibrium, the potential energy density function pertinent to the unit cell at $\boldsymbol{x}$ to within a harmonic approximation may be expressed as

$$
\Phi(\boldsymbol{x})=-\frac{1}{4 v} \sum_{\alpha} K_{i j}^{\alpha}\left(u_{i}\left(\boldsymbol{x}+\boldsymbol{R}_{\alpha}\right)-u_{i}(\boldsymbol{x})\right)\left(u_{j}\left(\boldsymbol{x}+\boldsymbol{R}_{\alpha}\right)-u_{j}(\boldsymbol{x})\right),
$$

The above relation was arrived based on the assumption that the unit cells consist of one atom, and so the summation over $\alpha$ represents the total potential energy at $\boldsymbol{x}$, accounting for the interactions between the atom located at $\boldsymbol{x}$ with every atom $\alpha$ located at $\boldsymbol{x}+\boldsymbol{R}_{\alpha}, \alpha=1,2, \ldots$ If the primitive unit cell of the crystal contains more than one atom, then the potential energy density of the unit cell is obtained by addition of the potential densities of all the atoms included in the unit cell.

Let $\dot{\boldsymbol{u}}(\boldsymbol{x})$ denote the velocity of the primitive unit cell at $\boldsymbol{x}$, and for simplicity assume to have one atom per unit cell. For a system occupying the entire three dimensional space and with a continuously distributed unit cells, the total potential energy and kinetic energy of the system, respectively, may be written as

$$
\begin{aligned}
& W=\int_{-\infty}^{\infty} \Phi(\boldsymbol{x}), \\
& K=\frac{1}{2} \int_{-\infty}^{\infty} \rho \dot{u}_{i} \dot{u}_{i},
\end{aligned}
$$

in which $\rho$ is the ratio of the mass of the atom in one primitive unit cell to its volume. By Taylor expansion of $\boldsymbol{u}\left(\boldsymbol{x}+\boldsymbol{R}_{\alpha}\right)$ about $\boldsymbol{x}$, one can write

$$
u_{i}\left(\boldsymbol{x}+\boldsymbol{R}_{\alpha}\right)-u_{i}(\boldsymbol{x})=\left.\frac{\partial u_{i}}{\partial x_{p}}\right|_{\boldsymbol{R}_{\alpha}=\mathbf{0}} R_{\alpha_{p}}+\left.\frac{1}{2} \frac{\partial^{2} u_{i}}{\partial x_{p} \partial x_{q}}\right|_{\boldsymbol{R}_{\alpha}=\mathbf{0}} R_{\alpha_{p}} R_{\alpha_{q}}
$$




$$
+\left.\frac{1}{6} \frac{\partial^{3} u_{i}}{\partial x_{p} \partial x_{q} \partial x_{r}}\right|_{\boldsymbol{R}_{\alpha}=\mathbf{0}} R_{\alpha_{p}} R_{\alpha_{q}} R_{\alpha_{r}}+\ldots
$$

By substituting Eq. (5.3) into Eq. (5.1) and employing the Hamilton's principle $\int_{t_{0}}^{t_{1}} \delta(W-K) d t=0$, the equations of motion for centrosymmetric crystals are obtained as

$$
\rho \ddot{u}_{i}=\tilde{C}_{i j m n} u_{j, m n}+\tilde{C}_{i j m n p q} u_{j, m n p q}+\tilde{C}_{i j m n p q r s} u_{j, m n p q r s}+\ldots,
$$

where "," in the subscript denotes differentiation with respect to $\boldsymbol{x}$; for example, $u_{j, m n}(\boldsymbol{x})=\partial^{2} u_{j}(\boldsymbol{x}) /\left(\partial x_{m} \partial x_{n}\right)$. The coefficients $\tilde{\boldsymbol{C}}$ appearing in the above relation depend on the atomic force constants and the equilibrium positions of the atoms as follows

$$
\begin{aligned}
\tilde{C}_{i j m n} & =-\frac{1}{2 v} \sum_{\alpha} K_{i j}^{\alpha} R_{\alpha_{m}} R_{\alpha_{n}}, \\
\tilde{C}_{i j m n p q} & =-\frac{1}{24 v} \sum_{\alpha} K_{i j}^{\alpha} R_{\alpha_{m}} R_{\alpha_{n}} R_{\alpha_{p}} R_{\alpha_{q}}, \\
\tilde{C}_{i j m n p q r s} & =-\frac{1}{72 v} \sum_{\alpha} K_{i j}^{\alpha} R_{\alpha_{m}} R_{\alpha_{n}} R_{\alpha_{p}} R_{\alpha_{q}} R_{\alpha_{r}} R_{\alpha_{s}} .
\end{aligned}
$$

The atomic force constants, $K_{i j}^{\alpha}$ are equivalent to the components of the Hessian matrix which are in turn equal to the value of the second derivative of the total potential energy with respect to the corresponding atomic positions at the equilibrium. The Hessian matrix is obtained by first principles density functional theory (DFT), and subsequently, the fourth, sixth, and eighth order constants given by relations (5.5) are evaluated. In the next section, the fourth and the higher order tensors pertinent to the second strain gradient elasticity will be related to the tensors 
$\tilde{C}$.

\subsection{Relations of bulk characteristic lengths and Lamé constants to $\tilde{\boldsymbol{C}}$}

As it was alluded to, formulation of surface elasticity in the context of second strain gradient theory and subsequent consideration of the film problem lead to several physical expressions relating the surface parameters, namely, surface stresses, surface energy, and surface elastic constants to second strain gradient parameters, namely, surface and bulk characteristic lengths in addition to the usual Lamé constants of the crystalline material. For an accurate determination of $\ell_{11}, \ell_{12}, \lambda$, and $\mu$ which are among the core parameters for the calculations of the above-mentioned physical entities, one should account for the electronic structure of the crystal. This can be achieved by relating the internal length-scales as well as Lamé constants to the tensors $\tilde{\boldsymbol{C}}$, calculated using first principles DFT as explained in the previous section. To this end, consider the most general form of the strain energy density function for centrosymmetric materials in the context of second strain gradient elasticity as

$$
\begin{aligned}
U_{0} & =\frac{1}{2} C_{i j k l} \epsilon_{i j} \epsilon_{k l}+F_{i j k l m n} \epsilon_{i j} \epsilon_{k l m n}+\frac{1}{2} G_{i j k l m n} \epsilon_{i j k} \epsilon_{l m n}+\frac{1}{2} I_{i j k l m n p q} \epsilon_{i j k l} \epsilon_{m n p q} \\
& +B_{i j k l}^{\circ} \epsilon_{i j k l} .
\end{aligned}
$$

With the aids of Eqs. (3.1), (3.5), (3.7), (3.9a), and (5.6), and in the absence of body forces, one can obtain the equations of motion as below

$$
\rho \ddot{u}_{i}=C_{i j k l} u_{k, l j}+\left(F_{p q j k l i}+F_{l i q j k p}-G_{k l i j q p}\right) u_{p, q j k l}+I_{j k l i m n r s} u_{s, m n r j k l} .
$$


From comparison of Eqs. (5.4) and (5.7), the following general relations are obtained

$$
\begin{aligned}
C_{i m j n} & =\tilde{C}_{i j m n}+\tilde{C}_{m j i n}-\tilde{C}_{m i j n} \\
\tilde{C}_{i j k l m n} & =\frac{1}{4}\left(F_{j k m n l i}+F_{j l m n k i}+F_{j m k n l i}+F_{j n m k l i}+F_{l i k m n j}+F_{k i l m n j}+F_{m i k l n j}\right. \\
& \left.+F_{n i k m l j}\right)-\frac{1}{6}\left(G_{n l i m k j}+G_{n k i m l j}+G_{k l i m n j}+G_{n m i l k j}+G_{m l i n k j}\right. \\
& \left.+G_{m k i n l j}\right), \\
\tilde{C}_{i j k l m n p q} & =\frac{1}{20}\left(I_{q k l i m n p j}+I_{q m l i k n p j}+I_{q n l i m k p j}+I_{q p l i m n k j}+I_{q k m i l n p j}+I_{q k n i m l p j}\right. \\
& +I_{q k p i m n l j}+I_{m k l i q n p j}+I_{n k l i m q p j}+I_{p k l i m n q j}+I_{q m n i k l p j}+I_{q m p i k l n j} \\
& +I_{q n p i m k l j}+I_{m k n i q l p j}+I_{m k p i q n l j}+I_{n k p i m q l j}+I_{m n l i q k p j}+I_{m p l i q n k j} \\
& \left.+I_{n p l i m q k j}+I_{m n p i q k l j}\right) .
\end{aligned}
$$

With the aids of comparison of Eqs. (3.13), (5.6), and (5.8), a set of equations for $\bar{a}-2 \bar{c}, \bar{a}^{\prime}-c_{3}, \bar{b}, \bar{b}^{\prime}, \lambda$, and $\mu$, pertinent to isotropic materials, in terms of the components of the tensors $\tilde{\boldsymbol{C}}$ is obtained. Subsequently, the bulk characteristic lengths are readily available via Eqs. (3.14).

\section{Numerical values for Lamé constants, bulk and surface characteristic lengths, modulus of cohesion, and surface and effective elastic con- stants of $\mathrm{Ag}, \mathrm{Au}$, and $\mathrm{Pt}$}

The evaluation of the surface characteristic length, $\ell_{10}$ and modulus of cohesion, $b_{0}$ for solids is at the stake of the availability of the experimental values of their 


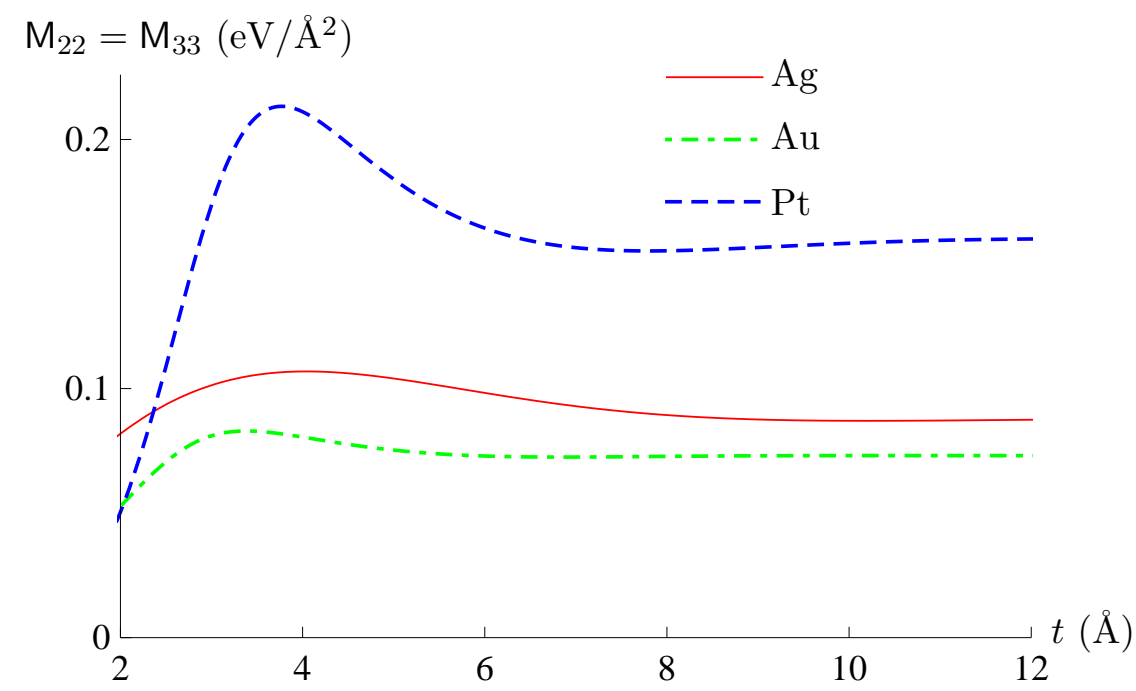

Figure 2: Residual surface stress, $\mathrm{M}_{22}=\mathrm{M}_{33}$ in units of $\mathrm{eV} / \AA^{2}$ versus the film thickness in units of $\AA$.

corresponding surface residual stress $\mathbf{M}$ and surface energy, $\gamma$. For this reason, the properties of interest associate with the fcc crystals, Ag, Au, and Pt are studied in this section. In particular, Lamé constants, bulk characteristic lengths, surface characteristic length, and modulus of cohesion of these elements are obtained via first principles DFT in conjunction with second strain gradient theory. Then, for Ag, $\mathrm{Au}$, and Pt films, one can go on to evaluate the surface elastic constants as well as the components of the effective elastic moduli tensor according to the formulations given in Sections 4.1 and 4.2, respectively.

The employed ab initio calculations are based on DFT (Hohenberg and Kohn, 1964; Kohn and Sham, 1965) incorporated in Vienna ab initio simulation package (VASP) (Kresse and Hafner, 1993, 1994a; Kresse and Furthmller, 1996a,b). This package employs the plane-wave basis set with ultrasoft pseudopotentials (Kresse and Hafner, 1994b). In all the calculations performed in this study, the kinetic energy cutoff is considered to be equal to $300 \mathrm{eV}$ and the Brillouin zone is meshed according to Monkhorst-Pack method (Monkhorst and Pack , 1976). The exchangecorrelation energy is estimated using the generalized gradient approximation (GGA) 


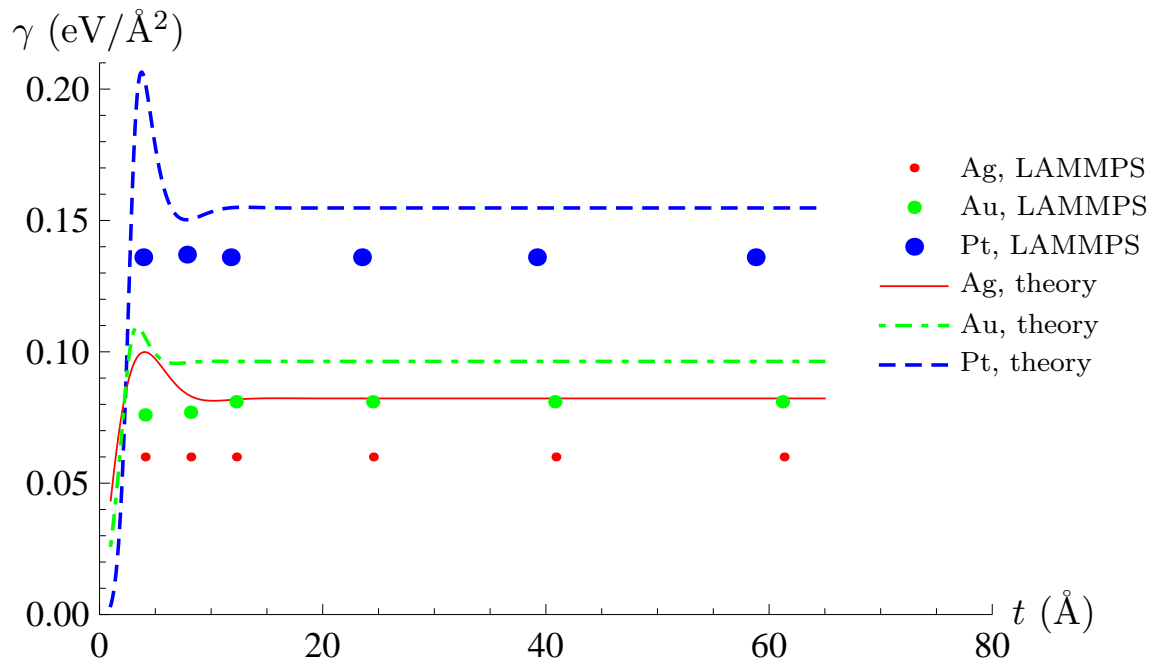

Figure 3: Surface energy, $\gamma$ in units of $\mathrm{eV} / \AA^{2}$ versus the film thickness in units of $\AA$.

through the Perdew-Burke-Ernzerhof (PBE) potential revised for solids (Perdew et al., 2008).

Based on the approach presented in Section 5 and the ab initio calculations, the values of Lamé constants in units of $\mathrm{eV} / \AA^{3}$ and bulk characteristic lengths in units of $\AA$ for Ag, Au, and Pt are calculated and summarized in Table 1. The values of Lamé constants calculated in the present work are in reasonable agreement with the pertinent experimental results (Freund and Suresh, 2003) which are given within the parentheses in Table 1.

According to the discussion in Section 4.3, by exploiting the experimental values for surface energy and residual surface stress tensor available in the literature, one can calculate the surface characteristic length, $\ell_{10}$ and modulus of cohesion, $b_{0}$. 
Table 1: Lamé constants in units of $\mathrm{eV} / \AA^{3}$ and bulk characteristic lengths in units of $\AA$ for $\mathrm{Ag}$, $\mathrm{Au}$, and Pt. The values for Lamé constants calculated in the present work are compared with the available experimental results at room temperature, given in parenthesis (Freund and Suresh, 2003).

\begin{tabular}{lcccccc}
\hline element & $\lambda\left(\mathrm{eV} / \AA^{3}\right)$ & $\mu\left(\mathrm{eV} / \AA^{3}\right)$ & $\ell_{11}(\AA)$ & $\ell_{12}(\AA)$ & $\ell_{21}(\AA)$ & $\ell_{22}(\AA)$ \\
\hline $\mathrm{Ag}$ & $0.56(0.58)$ & $0.24(0.29)$ & $0.91+1.03 i$ & $0.91-1.03 i$ & $1.37+1.66 i$ & $1.37-1.66 i$ \\
$\mathrm{Au}$ & $1.08(1.02)$ & $0.26(0.26)$ & $0.56+0.63 i$ & $0.56-0.63 i$ & $0.69+0.69 i$ & $0.69-0.69 i$ \\
$\mathrm{Pt}$ & $1.55(1.56)$ & $0.60(0.48)$ & $0.81+0.91 i$ & $0.81-0.91 i$ & $1.44+1.45 i$ & $1.44-1.45 i$ \\
\hline
\end{tabular}

Employing the experimental values for surface residual stress of $\mathrm{Ag}, \mathrm{Au}$, and $\mathrm{Pt}$ reported by Wassermann and Vermaak (1970), Mays et al. (1968), and Wassermann and Vermaak (1972), respectively, and the values of surface energy of these elements given by Tyson and Miller (1977), the modulus of cohesion and surface characteristic length of these elements are evaluated and summarized in Table 2. 


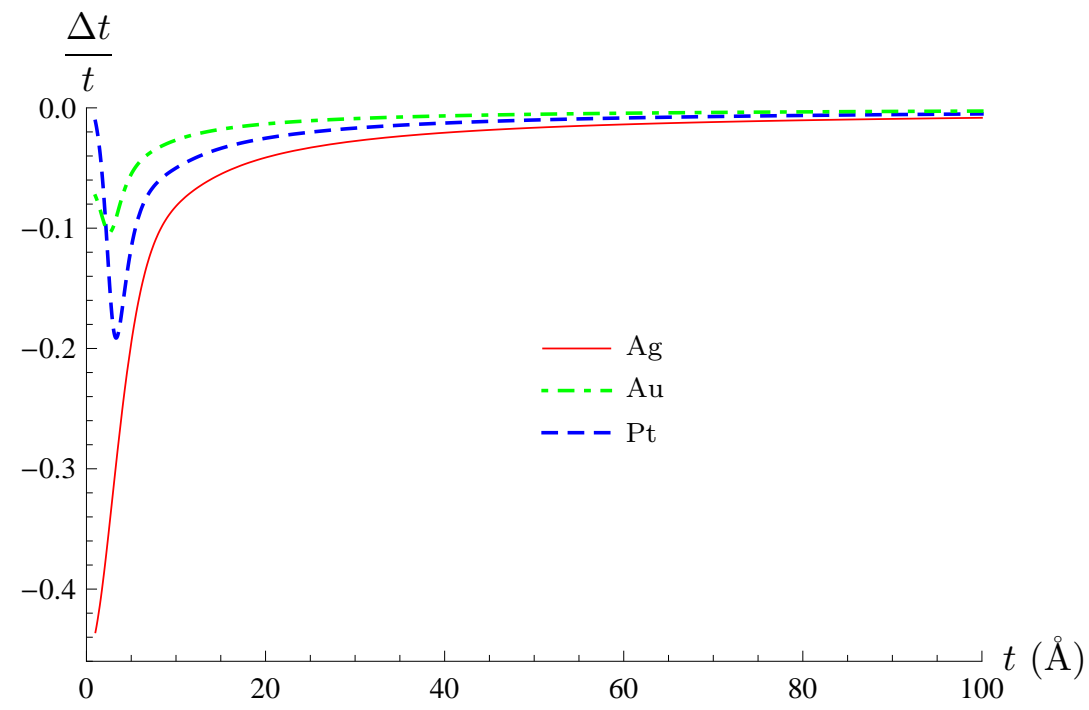

Figure 4: The normalized change of the film thickness, $\frac{\Delta t}{t}$ versus the film thickness in units of $\AA$.

Once the bulk material properties, $\lambda, \mu, \ell_{11}, \ell_{12}$, the additional parameter, $c_{1}$, surface characteristic length, $\ell_{10}$, and modulus of cohesion, $b_{0}$ are evaluated, the variation of residual surface stress, $M_{22}=M_{33}$, surface energy, $\gamma$, and the normalized change in film thickness, $\frac{\Delta t}{t}$ with the film thickness is obtained. Each of the Figs. 2, 3, and 4 displays the variation of, respectively, $\mathrm{M}_{22}=\mathrm{M}_{33}, \gamma$, and $\frac{\Delta t}{t}$ with thickness for three different elements of $\mathrm{Ag}, \mathrm{Au}$, and Pt. It is seen that for Table 2: Surface energy and surface residual stress in units of $\mathrm{eV} / \AA^{2}$, surface characteristic length in units of $\AA$, and modulus of cohesion in units of $\mathrm{eV} / \AA$ for $\mathrm{Ag}, \mathrm{Au}$, and Pt.

element $\quad$ surface energy $\left(\mathrm{eV} / \AA^{2}\right) \quad$ surface stress $\left(\mathrm{eV} / \AA^{2}\right) \quad \ell_{10}(\AA) \quad b_{0}(\mathrm{eV} / \AA)$

\begin{tabular}{lcccc}
\hline $\mathrm{Ag}$ & 0.082 & 0.088 & $2.16 i$ & -1.87 \\
$\mathrm{Au}$ & 0.096 & 0.073 & $0.259+0.420 i$ & $-0.157+0.314 i$ \\
$\mathrm{Pt}$ & 0.155 & 0.160 & $0.271+0.936 i$ & $-0.984+0.622 i$ \\
\hline
\end{tabular}




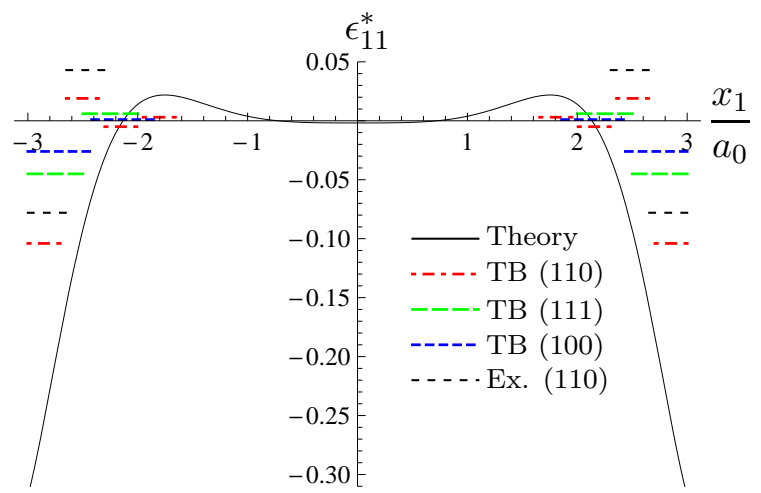

(a)

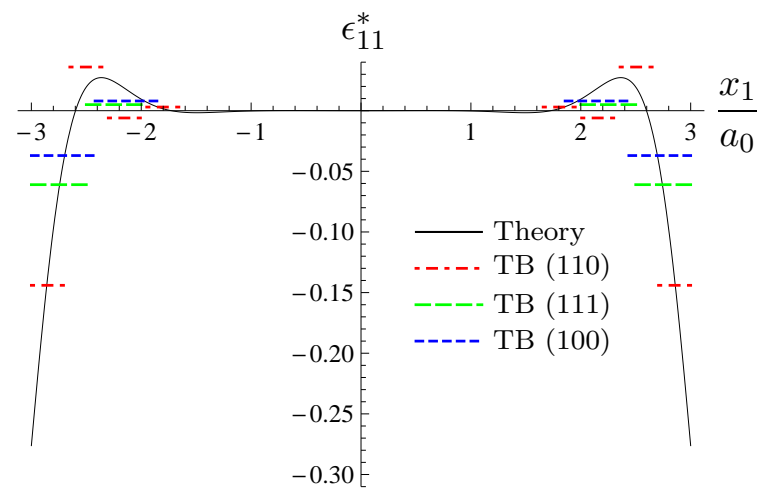

(b)

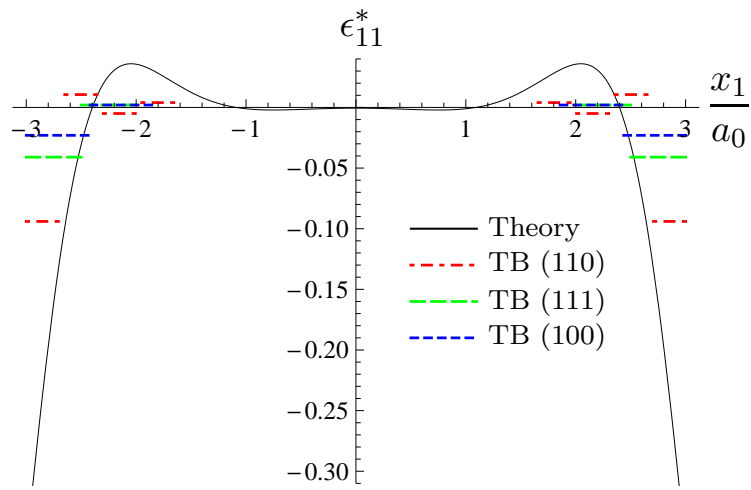

(c)

Figure 5: Surface relaxation delineated by strain component, $\epsilon_{11}^{*}$ versus normalized coordinate $x_{1} / a_{0}$ for (a) Ag, (b) Au, and (c) Pt; film thickness is assumed to be $6 a_{0}$. The available values of the average strain computed via tight-binding scheme (Rosato et al., 1989) as well as the experimental data measured via high-energy ion scattering (Kuk and Feldman, 1984) are plotted for comparison.

these crystals the maximum value of the residual stress, $M_{22}=M_{33}$ occurs for the film thickness of about $4 \AA$. For thicknesses beyond about $8 \AA$, the variations associated with $\mathrm{Ag}, \mathrm{Au}$, and $\mathrm{Pt}$, respectively, reach their corresponding experimental plateaus of $0.088,0.073$, and 0.160 in units of $\mathrm{eV} / \AA^{2}$; the experimental values are due to Wassermann and Vermaak (1970), Mays et al. (1968), and Wassermann and Vermaak (1972), respectively. Furthermore, Surface energy associated to each element $\mathrm{Ag}, \mathrm{Au}$, and $\mathrm{Pt}$ is also calculated using the package LAMMPS with embedded atom potentials and depicted in Fig. 3. It is noteworthy to mention that due to the fact that the bulk characteristic lengths are complex numbers (Table 1), the varia- 


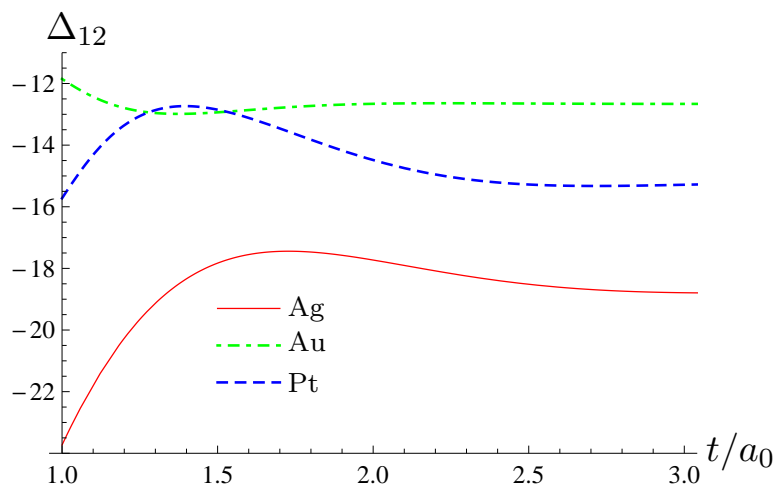

(a)

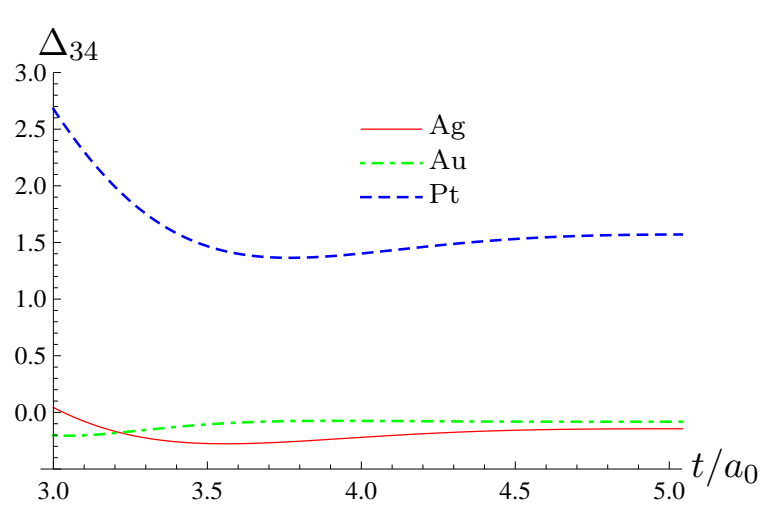

(c)

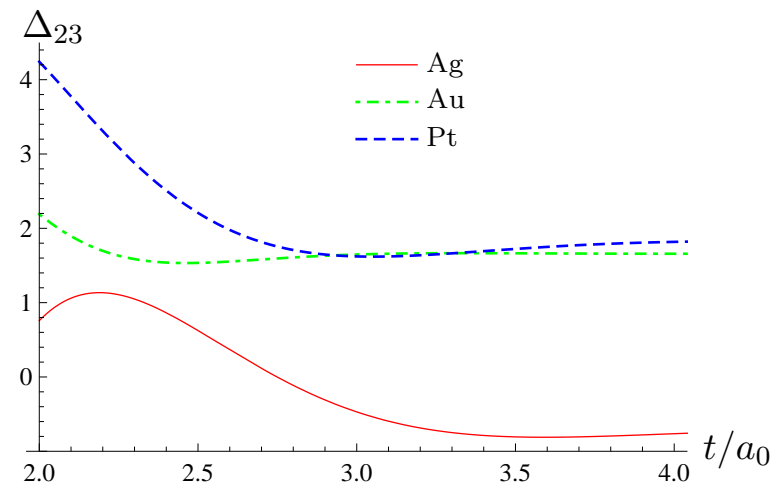

(b)

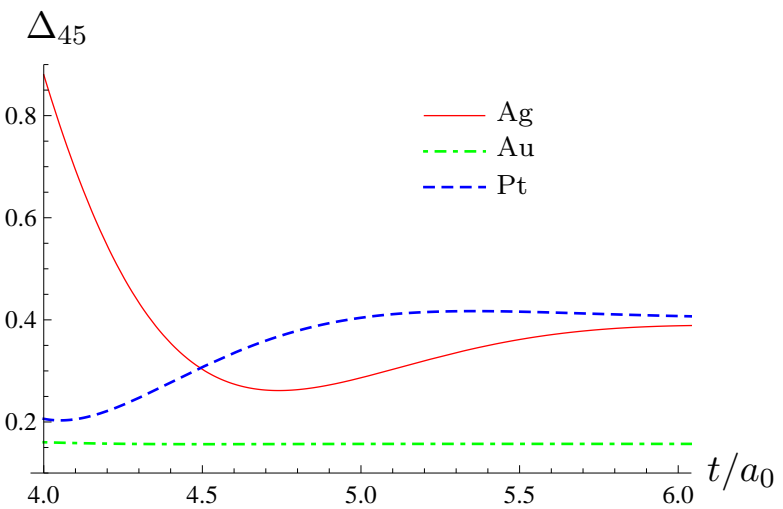

(d)

Figure 6: The normalized inter-layer relaxation (a) $\Delta_{12}$, (b) $\Delta_{23}$, (c) $\Delta_{34}$, and (d) $\Delta_{45}$ with respect to the inter-layer distance in percent versus the normalized film thickness $t / a_{0}$.

tions of the surface residual stress, surface energy, and change in film thickness are oscillatory which damp rapidly as the film thickness increases. Rosato et al. (1989) who employed a simple TB model have reported oscillatory relaxation of surfaces for fcc transition metals; they found satisfactory agreement between their theoretical results and those of the available laboratory experiments. Rosato et al. (1989) have compared the data from their simulations with the available experimental results in Table 3 of their paper. From this table it is evident that the oscillatory relaxation of surfaces, in all the reported cases, incur with the contraction of the first inter-layer followed by dilation of the second inter-layer and so on. The amplitude of the absolute value of the contraction in the first inter-layer is the largest and decreases with distance from the surface. The trend of their data reveals that the ratio of the net 
change of the inter-layer thicknesses to the total thickness of the film decays with increasing number of atomic inter-layers within the film. To the end of comparison of the results obtained from the present theory with the computational and experimental results, the variation of $\epsilon_{11}^{*}$ through the depth of the film is plotted against the normalized coordinate $x_{1} / a_{0}$ for a film of thickness $t=6 a_{0}$ in Figs. 5(a)-(c) for Ag, $\mathrm{Au}$, and Pt, respectively. The presented experimental results for Ag were obtained by Kuk and Feldman (1984) employing the high-energy ion scattering. It is observed that the results of the present continuum theory are in reasonable agreement with the available computational and experimental data. Moreover, using the current theoretical approach, one can determine the inter-layer relaxations as a function of film thickness, as shown in Figs. 6(a)-(d). In these figures, $\Delta_{i j}$ is the normalized relaxation between the atomic layers $i$ and $j$ with respect to the inter-layer distance given in percentage. In these plots, the inter-layer distances are assumed equal to the average value of about $0.5 a_{0}$.

As explained in Section 4, the top and bottom surfaces of an infinitely extended planar film, after relaxation, remain flat and their pertinent curvature tensors are zero. Figs. 7(a) and (b) compare the deformed configurations for Ag films of threeatomic-layer thick of infinite extent and finite extent obtained by employing the package LAMMPS; there are no external loadings and the observed deformations are solely due to the surface effects. It is observed that in contrast to the film of finite extent, the infinitely extended film has no curvature on its surfaces. From Fig. 7(b), 


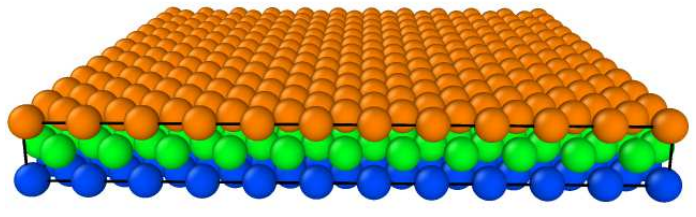

(a)

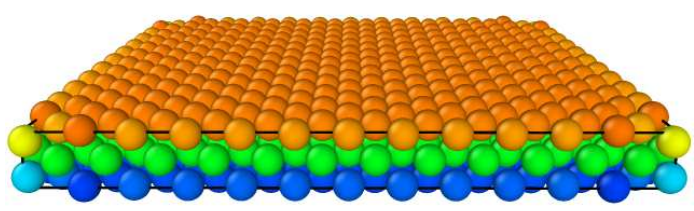

(b)

Figure 7: Modeling of (a) infinite and (b) finite extent Ag films consisting of three-atomic-layers via LAMMPS.

it is evident that associated with the Ag film of finite extent there is a mild gradation of curvature from the center of the top surface towards the edges. Another important parameter in the context of the present work is the surface elastic constants given according to Eqs. (4.15). Figs. 8(a), (b), and (c) show, respectively, the variations of the components of the surface elastic constants, $C_{2222}, C_{2233}$, and $C_{2323}$ in units of $\mathrm{eV} / \AA^{2}$ with the thickness of the films in units of $\AA$; three different variations pertinent to $\mathrm{Ag}, \mathrm{Au}$, and $\mathrm{Pt}$ films are displayed in each figure. The absolute values of these surface parameters attain their maximum for the film thickness of about 4 $\AA$. As $t$ becomes large, the surface parameters approach the values they have when the two surfaces of the film are far apart and don't interact.

In continue, by employing the discussions of Section (4.2), one can determine the components of the effective elastic constants of the film in terms of its thickness. Each of the Figs. 9(a)-(e) provides the indicated normalized component of the effective elastic moduli for $\mathrm{Ag}, \mathrm{Au}$, and Pt films.

As mentioned previously, since the bulk characteristic lengths are complex numbers (Table 1), the variations of the surface elastic constants and effective elastic constants of the film are oscillatory which damp rapidly as the film thickness increases. As it is observed from Figs. 8 and 9, for ultra-thin films with small enough thicknesses the size dependent character of surface elastic parameters as well as 
(a)

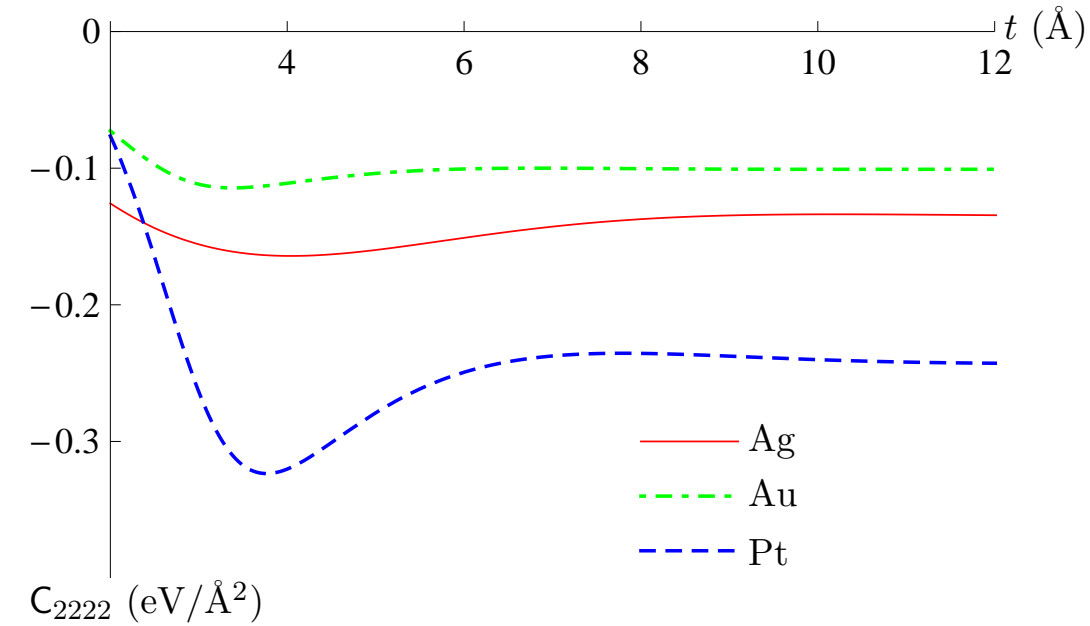

(b)

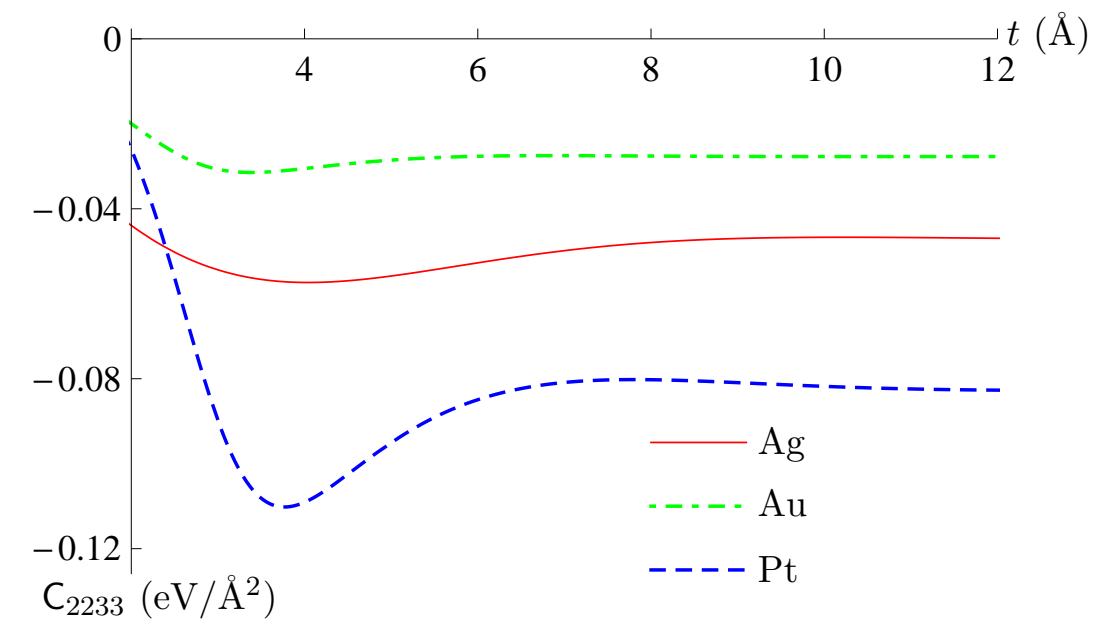

(c)

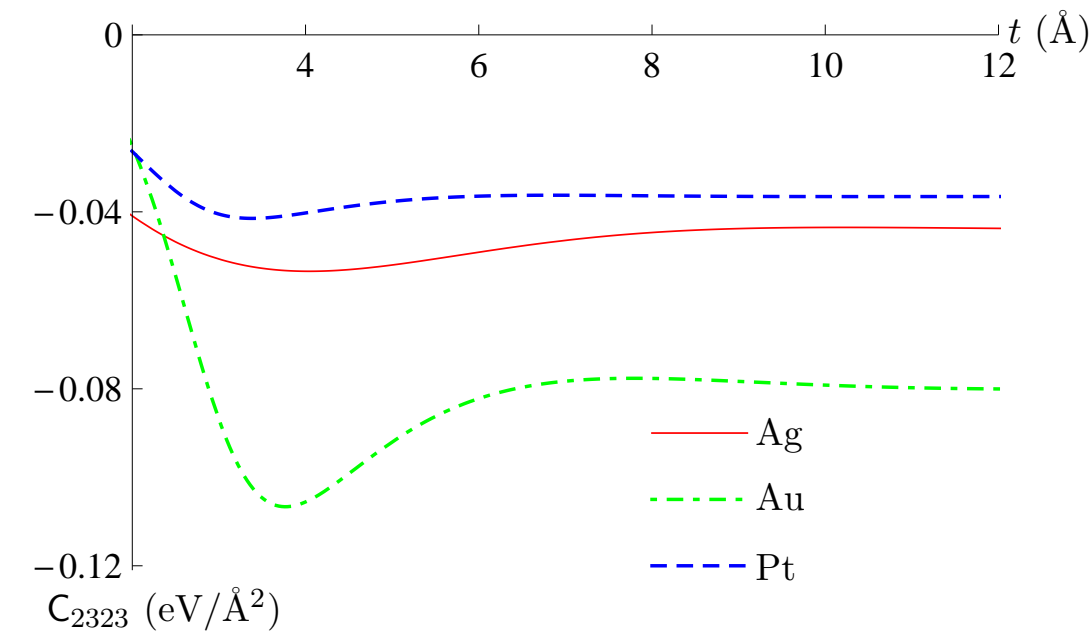

Figure 8: The components of surface elastic constants, (a) $C_{2222}$, (b) $C_{2233}$, and (c) $C_{2323}$ in units of $\mathrm{eV} / \AA^{2}$ versus the thickness of the film in units of $\AA$. 


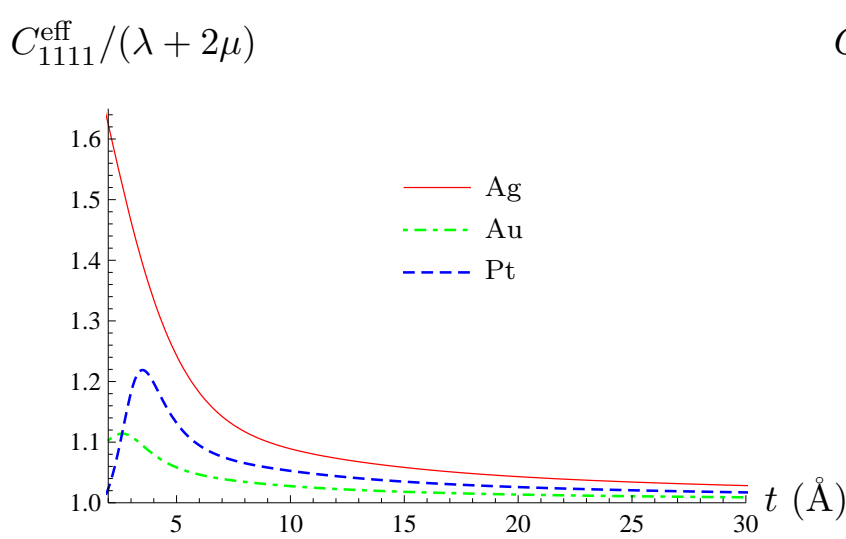

(a)

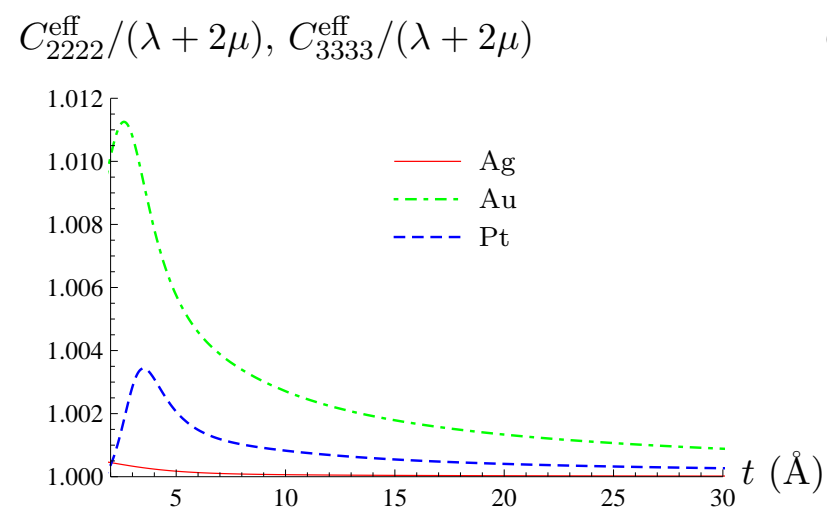

(c)

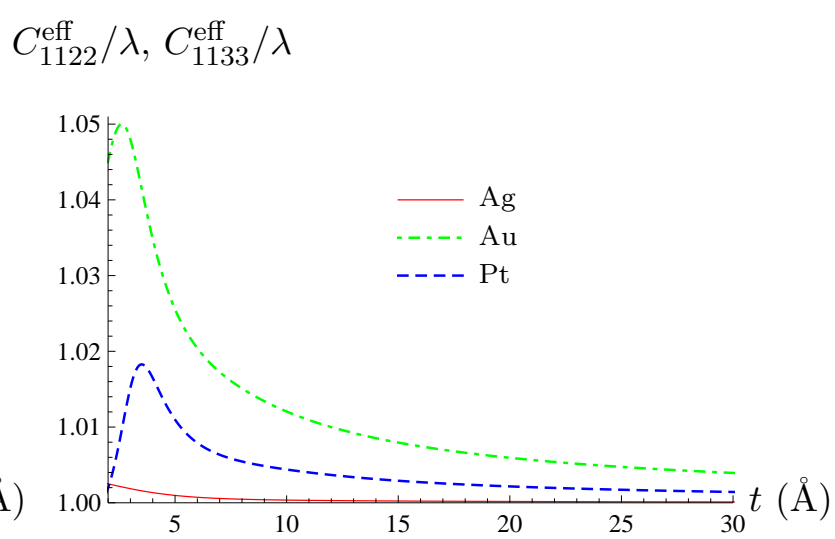

(b)

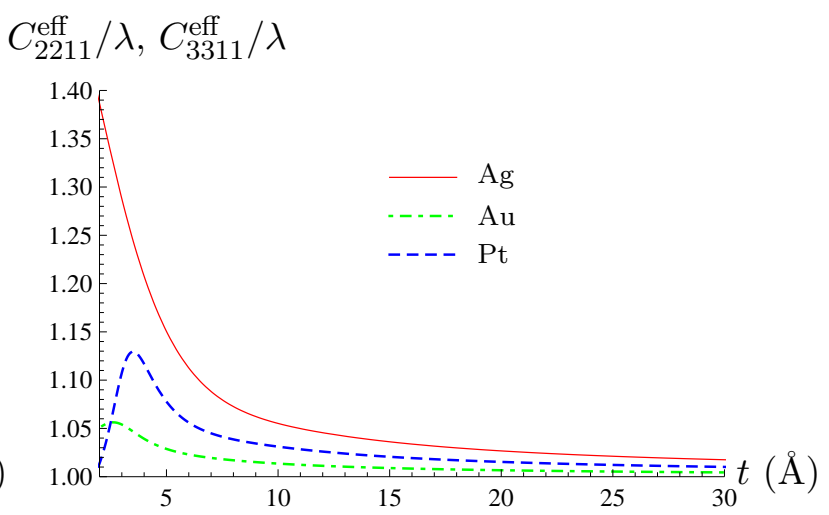

(d)

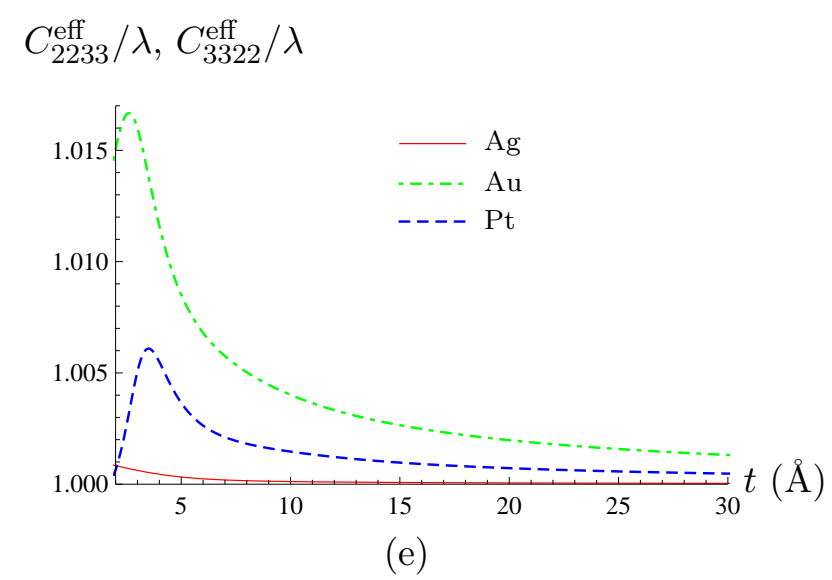

Figure 9: (a)-(e) The components of the normalized effective elastic constants, $\boldsymbol{C}^{\text {eff }}$ versus the thickness of the film in units of $\AA$ for $\mathrm{Ag}, \mathrm{Au}$, and $\mathrm{Pt}$.

effective elastic constants is captured. 


\section{Conclusion}

Second strain gradient theory was given by Mindlin (1965) mainly in an effort to account for the surface effects which could not be detected via Mindlin's first strain gradient theory. Gurtin and Murdoch (1975) who developed surface elasticity theory account for the surface effect from a completely different viewpoint. In surface elasticity where consideration of the equilibrium conditions for the bulk and surface gives rise to two sets of equations - one set of equations for the bulk material and another set for its complementary surface material - three surface parameters, namely, tangential surface strain tensor, surface stress tensor, and surface elastic modulus tensor reveal. On the other hand, the mathematical framework of second strain gradient theory gives rise to a surface characteristic length as well as modulus of cohesion; Mindlin (1965) and Ojaghnezhad and Shodja (2013) showed that modulus of cohesion is expressible in terms of surface energy, surface and bulk characteristic lengths, and Lamé constants. The determination of all the above-mentioned parameters has posed serious challenges, both experimentally and theoretically. Moreover, a valid concern is whether there are some physically sensible relationships between the hereditary parameters of these two frameworks, which were both primarily proposed to capture the surface effect. To fulfill this concern, the present work revisits surface elasticity in the context of second strain gradient theory. The reformulations in the new framework lead to two new key equations; one for the net surface stress on the boundary which is instrumental in relating the surface parameters in surface 
elasticity to the Mindlin's gradient theory parameters, and another for the total elastic energy which aids finding an expression for the surface energy. In continue, an estimate for the effective elastic constants is presented. Furthermore, the current work provides a theoretical remedy for the calculations of Lamé constants, surface and bulk characteristic lengths, modulus of cohesion, surface elastic constants, and effective elastic constants. First principles DFT is used to calculate Lamé constants and bulk characteristic lengths of crystalline solids. Having the values of Lamé constants and bulk characteristic lengths, then by exploiting the experimental values for surface energy and surface residual stress tensor available in the literature, one can evaluate the surface characteristic length and modulus of cohesion using the pertinent closed-form expressions derived in this article. In continue, the components of surface elastic modulus tensor and effective elastic constants of thin film are computed for different thicknesses of films made of fcc crystals, Ag, Au, and Pt.

\section{Acknowledgement}

The authors acknowledge the support of Iran National Science Foundation (INSF).

Agiasofitou, E.K. and Lazar, M., 2009. Conservation and balance laws in linear elasticity of grade three. J. Elast. Vol. 94, 69-85.

Brand, L., 1966. Vector and tensor analysis. John Wiley \& Sons, Inc. New York.

Cammarata, R.C. and Sieradzki, K., 1989. Effects of surface stresses on the elastic moduli of thin films and superlattices. Phys. Rev. Lett. Vol. 62(17), 2005-2008. 
Cauchy, A.L., 1851. Note sur l'rquilibre et les mouvements vibratoires des corps solides. Comptes-Rendus 32, 323-326.

Finnis, M.W. and Sinclair, J.E., 1984. A simple empirical N-body potential for transition metals. Philos. Mag. A 50, 4555.

Freund, L.B. and Suresh, S., 2003. Thin film materials, Stress, defect formation, and surface evolution. Cambridge university press.

Gurtin, M.E., 1995. The nature of configurational forces. Arch. Rat. Mech. Anal. Vol. 131, 67-100.

Gurtin, M.E. and Murdoch, A.I., 1974. A continuum theory of elastic material surfaces. Arch. Rat. Mech. Anal. Vol. 57, 291-323.

Hohenberg, P. and Kohn, W., 1964. Inhomogeneous electron gas. Phys. Rev. 136, B864B871.

Javili, A., dell'sola, F., and Steinmann, P., 2013. Geometrically nonlinear highergradient elasticity with energetic boundaries. J. Mech. Phys. Solids. Vol. 61, 23812401.

Kohn, W. and Sham, L., 1965. Self-consistent equations including exchange and correlation effects. Phys. Rev. 140, A1133A1138.

Kresse, G. and Furthmller, J., 1996a. Efficiency of ab-initio total energy calculations 
for metals and semiconductors using a plane-wave basis set. Comput. Mater. Sci. Vol. 6, 1550 .

Kresse, G. and Furthmller, J., 1996b. Efficient iterative schemes for ab-initio totalenergy calculations using a plane-wave basis set. Phys. Rev. B 54, 1116911186.

Kresse, G. and Hafner, J., 1993. Ab-initio molecular dynamics for liquid metals. Phys. Rev. B 47, 558561.

Kresse, G. and Hafner, J., 1994a. Ab-initio molecular dynamics simulation of the liquidmetal-amorphous-semiconductor transition in germanium. Phys. Rev. B 49, 1425114269.

Kresse, G. and Hafner, J., 1994b. Norm-conserving and ultrasoft pseudopotentials for first-row and transition elements. J. Phys.: Condens. Matter 6, 82458257.

Kuk, Y. and Feldman, L.C., 1984. Oscillatory relaxation of the $\mathrm{Ag}(110)$ surface. Phys. Rev. B 30(10), 5811-5816.

Mays, C.W., Vermaak, J.S., Kuhlmann-Wilsdorf, D., 1968. On surface stress and surface tension: II. Determination of the surface stress of gold. Surf. Sci. Vol. $12(2), 134-140$.

Mindlin, R.D., 1965. Second gradient of strain and surface-tension in linear elasticity. Int. J. Solids Struc. Vol. 1, 417-438. 
Mindlin, R.D., Eshel, N.N., 1968. On first strain-gradient theories in linear elasticity. Int. J. Solids Struc. Vol. 4, 109-124.

Mindlin, R.D., 1972. Elasticity, piezoelectricity and crystal lattice dynamics. J. Elast. Vol. 2(4), 217-282.

Monkhorst, H. and Pack, J., 1976. Special points for Brillouin-zone integrations. Phys. Rev. B 13, 51885192.

Ojaghnezhad, F. and Shodja H.M., 2012. A combined first principles and analytical treatment for determination of the surface elastic constants: application to $\mathrm{Si}(001)$ ideal and reconstructed surfaces. Phil. Mag. Lett. Vol. 92, 7-19.

Ojaghnezhad, F. and Shodja H.M., 2013. A combined first principles and analytical determination of the modulus of cohesion, surface energy, and the additional constants in the second strain gradient elasticity. Int. J. Solids Struc. Vol. 50, $3967-3974$.

Perdew, J.P., Ruzsinszky, A., Csonka, G.I., Vydrov, O.A., Scuseria, G.E., Constantin, L.A., Zhou, X., and Burke, K., 2008. Restoring the density-gradient expansion for exchange in solids and surfaces. Phys. Rev. Lett. Vol. 100, 136406.

Polizzotto, C., 2013a. A second strain gradient elasticity theory with second velocity gradient inertia Part I: Constitutive equations and quasi-static behavior. Int. J. Solids Struct. Vol. 50, 3749-3765. 
Rosato, V., Guillope, M., Legrand, B., 1989. Thermodynamical and structural properties of f.c.c. transition metals using a simple tight-binding model. Phil. Mag. A. Vol. 59(2), 321-336.

Shodja, H.M., Ahmadpoor, F., Tehranchi, A., 2012. Calculation of the additional constants for fcc materials in second strain gradient elasticity: behavior of a nano-size BernoulliEuler beam with surface effects. J. Appl. Mech. Vol. 79, 021008021015.

Shodja, H.M., Tehranchi, A., 2010. A formulation for the characteristic lenghts of fcc materials in first strain gradient elasticity via the Sutton-Chen potential. Philos. Mag. Vol. 90, 18931913.

Shodja, H.M., Tehranchi, A., 2012. A formulation for the characteristic lenghts of fcc materials in first strain gradient elasticity via the Sutton-Chen potential. Corrigendum Philos. Mag. Vol. 92, 11701171.

Shodja, H.M., Zaheri, A., Tehranchi, A., 2013. Ab initio calculations of characteristic lengths of crystalline materials in first strain gradient elasticity. Mech. Mater. Vol. $61,7378$.

Shuttleworth, R., 1950. The surface tension of solids. Proc. Phys. Soc. A 63, 444-457.

Simha, N.K., Bhattacharya, K., 1997. Equilibrium conditions at corners and edges of an interface in a multiphase solid. Mat. Sci. Eng. A238, 32-41. 
Simha, N.K., Bhattacharya, K., 1998. Kinetics of phase boundaries with edges and junctions. J. Mech. Phys. Solids. Vol. 46, 2323-2359.

Simha, N.K., Bhattacharya, K., 2000. Kinetics of phase boundaries with edges and junctions in a three-dimensional multi-phase body. J. Mech. Phys. Solids. Vol. 48, 2619-2641.

Toupin, R.A., Gazis, D.C., 1964. Surface effects and initial stress in continuum and lattice models of elastic crystals. in: Proceedings of the International conference on lattice dynamics. Wallis (ed.), Copenhagen, August, 1963. Pergamon press. pp. 597-602.

Tyson, W.R., Miller, W.A., 1977. Surface free energies of solid metals: estimation from liquid surface tension measurements. Surf. Sci. Vol. 62, 267-276.

Voigt, W., 1887. Theoretische studien ber die elasticittsverhltnisse der krystalle. Abh. Kgl. Ges. Wiss. Gttingen Vol. 34, 3-51.

Voigt, W., 1894. ber Median ohne innere Krfte und eine durch sie gelieferte mechanische deutung der Maxwell-Hertzschen gleichungen. Abh. Kniglichen Gesellschaft Wiss. Gttingen Vol. 40, 72-79.

Wassermann, H.J., Vermaak, J.S., 1970. On the determination of a lattice contraction in very small silver particles. Surf. Sci. Vol. 22, 164-172. 
Wassermann, H.J., Vermaak, J.S., 1972. On the determination of the surface stress of copper and platinum. Surf. Sci. Vol. 32, 168-174. 